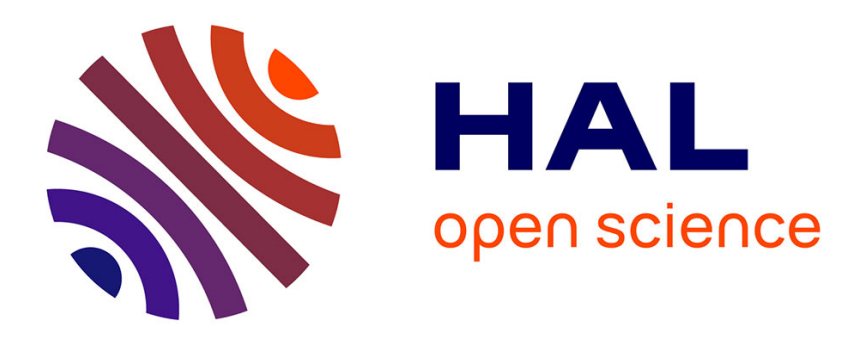

\title{
Non identical coupled networks with a geographical model for human behaviors during catastrophic events Guillaume Cantin
}

\section{To cite this version:}

Guillaume Cantin. Non identical coupled networks with a geographical model for human behaviors during catastrophic events. 2017. hal-01673706

\author{
HAL Id: hal-01673706 \\ https://hal.science/hal-01673706
}

Preprint submitted on 31 Dec 2017

HAL is a multi-disciplinary open access archive for the deposit and dissemination of scientific research documents, whether they are published or not. The documents may come from teaching and research institutions in France or abroad, or from public or private research centers.
L'archive ouverte pluridisciplinaire HAL, est destinée au dépôt et à la diffusion de documents scientifiques de niveau recherche, publiés ou non, émanant des établissements d'enseignement et de recherche français ou étrangers, des laboratoires publics ou privés. 


\title{
NON IDENTICAL COUPLED NETWORKS WITH A GEOGRAPHICAL MODEL FOR HUMAN BEHAVIORS DURING CATASTROPHIC EVENTS
}

\author{
Guillaume CANTIN
}

Laboratoire de Mathématiques Appliquées du Havre

Normandie Univ, FR CNRS 3335, ISCN, 25 rue Philippe Lebon 76600 Le Havre, France

guillaumecantin@mail.com

\begin{abstract}
The purpose of this work is to study coupled networks of non identical instances of the PCR system (Panic-Control-Reflex), which is a geographical model for human behaviors during catastrophic events. We endow the subsequent graph with superposed linear and quadratic couplings, and explore the effect of the topology of the network on the dynamics of each node. Especially, we investigate the possibility of controlling the panic level in the network by a clever disposal of the connections. We establish a necessary and sufficient condition for synchronization, without any reductive assumption on the nature of the network, and study the global stability of the trivial equilibrium. We illustrate our theoretical results by numerical simulations of randomly generated networks.
\end{abstract}

Keywords. PCR system; coupled network; global stability; synchronization.

\section{$\S 1 \quad$ Introduction}

In this paper, we aim to present the continuation of a recent work [5], concerning the PCR system (Panic-Control-Reflex), which is a geographical model for human behaviors during catastrophic events, established in 2013 in order to better understand and predict behavioral reactions of individuals facing a brutal disaster, which can have a natural or an industrial origin [21, 27, 22]. In this previous work, a complete stability and bifurcation analysis of that dynamical system was proposed, exhibiting a degeneracy case of a saddle-node bifurcation at infinity, with two possible asymptotic behaviors for the solutions. The first possible asymptotic behavior corresponds to a favorable return of all individuals affected by the catastrophe to a daily way of life, while the second possible behavior of the solutions presents a persistence of panic.

The present paper is devoted to the study of coupled networks of non identical PCR systems, with the concern of modeling the geographical relief of the area impacted by the catastrophe, as well as the individuals displacements and contacts. In a recent conference paper [6], a concrete example prepared with the collaboration of geographers, concerning the particular risk of tsunami on the Mediterranean coast, has been studied. It is shown in this latter article that the evacuation of high risk zones corresponding to the beach places, towards the refuge zones situated in the city center, plays a very decisive role. For instance, a plugged corridor can provoke a persistence of panic. At the opposite, an additional evacuation path from the beach towards the city-center can help individuals return to the daily behavior. For the sake of generality, we aim to prove for any abstract PCR network that a lightened organization of the connections, on the disaster zone, is a crucial key for a global return of the affected population to a daily behavior. Indeed, we shall first investigate patterns emerging from basic configurations, before looking ahead to larger networks, since it is a subtle question to relate the global dynamic of the network to the internal dynamic of each of its cells. The generality of our study gives a rigorous framework for better understanding the impact of the connections disposal in any situation concerned with our model, and it might be adapted for various non identical coupled networks.

A huge literature tackles the subject of coupled networks, with a mathematical approach. Many questions are discussed, such as synchronization of chaotic systems or oscillators [8, 19, 4, 3, 2], bifurcation analysis and global normal form calculation [12, 23]. Different topologies are considered, with symmetry or orientation assumptions. Many applications to physical or biological phenomena are studied, such as electric circuits networks or neural networks [25, 30, 29, 24]. 
Our analysis of PCR systems networks will deal with non identical nodes coupled networks, with an asymmetric topology of variable density, built with the aim to model the variety of human behavioral reactions, according to the nature of the disaster, and to the geographical relief of the impacted zone. As a tool, we will use graph theory path finding algorithms [10, 11], yet it is not the subject of our analysis.

The outline of the paper is the following. We shall begin with preliminaries about non identical coupled networks. After recalling some basics about the PCR system, we shall define PCR networks and study two-nodes configurations, with a linear or quadratic coupling form. Our main result is presented in the third section, and establishes a link between on the first hand the possible evacuation of panic nodes, that can be detected using graph theory algorithms, and on the other hand the research of a global return to a daily behavior among the considered population. The global stability of the trivial equilibrium will be analyzed in the general case, without any reductive assumption on the topology of the network. Finally, we shall present numerical simulations in order to illustrate our qualitative results, with randomly generated PCR networks.

\section{$\S 2 \quad$ Non identical coupled networks}

\subsection{Preliminaries}

Let us consider a dynamical system

$$
\dot{x}=f(t, x, \lambda), \quad t \geq t_{0}, \quad x \in \Omega \subset \mathbb{R}^{n}, \quad \lambda \in \mathbb{R}^{p},
$$

where $n$ and $p$ are positive integers, $\Omega$ is a subset of $\mathbb{R}^{n}, f$ is a smooth function defined on $\mathbb{R} \times \Omega \times \mathbb{R}^{p}$, and $t_{0}$ is a given initial time. We suppose that the system (1) admits a bifurcation at $\lambda_{0}$, that is a change in the stability and structure of its invariant sets for $\lambda\left\langle\lambda_{0}\right.$ and for $\left.\lambda\right\rangle \lambda_{0}$ (see [16] for instance, or [17] for the case of non autonomous systems).

Next, we consider a graph $\mathscr{G}$ formed with a finite set $\mathscr{V}$ of $N$ vertices $\{1, \ldots, N\}(N \geq 2)$, and two finite sets $\mathscr{E}_{L}$ and $\mathscr{E}_{Q}$ of oriented edges, corresponding to linear and quadratic couplings respectively. An element $(i, j)$ of $\mathscr{E}_{L}$ or $\mathscr{E}_{Q}$ corresponds to a directed connection from vertex $i$ towards vertex $j$. For short, we will name the elements of $\mathscr{E}_{L}$ and $\mathscr{E}_{Q}$ linear edges and quadratic edges respectively. We associate to each vertex $i \in \mathscr{V}$ an instance of system (1) with one parameter value $\lambda_{i}$, and consider a coupled network of the form

$$
\dot{x}_{i}=f\left(t, x_{i}, \lambda_{i}\right)+\sum_{j=1}^{N} L_{i, j}(t) \mathcal{H} x_{j}+\sum_{j=1}^{N}(Q(t, X))_{i, j}, \quad 1 \leq i \leq N
$$

where $x_{i} \in \mathbb{R}^{n}$ is the vector representing the state of vertex $i$ and $X=\left(x_{1}, \ldots, x_{N}\right)^{T}$. Here, we consider the matrix of linear connectivity $L(t)=\left(L_{i, j}(t)\right)$, which is symmetric if the linear edges of the graph are non oriented. More precisely, if $(i, j) \in \mathscr{E}_{L}, i \neq j$, we have $L_{j, i}(t)>0$, for all $t \geq t_{0}$. If $(i, j) \notin \mathscr{E}_{L}, i \neq j$, we have $L_{j, i}(t)=0$, for all $t \geq t_{0}$. Moreover, the diagonal elements of matrix $L$ satisfy

$$
L_{i, i}(t)=-\sum_{\substack{j=1 \\ j \neq i}}^{N} L_{j, i}(t)
$$

thus $L(t)$ is a zero column sum matrix. The matrix $\mathcal{H}$ is of order $n$, and determines which components of vertices $x_{i}$ and $x_{j}$ are coupled.

Similarly, we define the matrix of quadratic connectivity $Q(t, X)$ as follows. If $(i, j) \in \mathscr{E}_{Q}$, $i \neq j$, we set

$$
(Q(t, X))_{j, i}=-Q_{j, i}(t) \kappa\left(x_{i}, x_{j}\right), \quad(Q(t, X))_{i, j}=+Q_{j, i}(t) \kappa\left(x_{i}, x_{j}\right),
$$

where $Q_{j, i}(t)$ is a smooth real valued function of $t$ with positive values. The function $\kappa$ is defined on $\mathbb{R}^{n} \times \mathbb{R}^{n}$ by a quadratic expression

$$
\kappa\left(x_{i}, x_{j}\right)=\left(\sum_{1 \leq \alpha, \beta \leq n} \kappa_{l}^{\alpha, \beta} x_{i}^{(\alpha)} x_{j}^{(\beta)}\right)^{T}, \quad 1 \leq l \leq n
$$


where $x_{i}^{(\alpha)}, 1 \leq \alpha \leq n$ denote the components of $x_{i}$, and $\kappa_{l}^{\alpha, \beta} \in \mathbb{R}$ for all $l, \alpha$ and $\beta$ such that $1 \leq l \leq n, 1 \leq \alpha \leq n$ and $1 \leq \beta \leq n$. The matrix of coefficients $\left(Q_{j, i}\right)$ is of order $N$ and anti symmetric. We precise that the function $\kappa$ is not necessarily symmetric.

For convenience, we introduce the function $\mathscr{Q}(t, X)$, defined as a $\mathbb{R}^{n \times N}$ vector valued function, by setting

$$
\mathscr{Q}(t, X)=\left(\mathscr{Q}_{i}(t, X)\right)^{T}=\left(\sum_{j=1}^{N}(Q(t, X))_{i, j}\right)^{T}, \quad 1 \leq i \leq n .
$$

In the sequel, we will omit the time dependence of coefficients in matrices $L$ and $Q$, in order to lighten our notations. Furthermore, we will assume that there exists a minimum coupling strength $\eta_{0}>0$ such that

$$
L_{i, j}(t) \geq \eta_{0}, \quad Q_{i, j}(t) \geq \eta_{0},
$$

for all $t \geq t_{0}$ and $i, j$ such that $1 \leq i, j \leq n, L_{i, j}(t) \neq 0$ or $Q_{i, j}(t) \neq 0$.

We will refer to the vertices of $\mathscr{G}$ with the word nodes. The term $f\left(t, x_{i}, \lambda_{i}\right)$ determines the internal dynamic of each node, while the coupling terms contained in $L$ and $Q(X)$ depend on the topology of the network. For convenience and clarity, we will associate to each parameter value $\lambda_{i}$ one color. Two nodes $x_{i}$ and $x_{j}$ of the same color will then admit the same parameter value, that is $\lambda_{i}=\lambda_{j}$, thus the same internal dynamic.

Example 1. Assume $n=2, N=4$, and consider the graph $\mathscr{G}$ depicted in Figure 1.

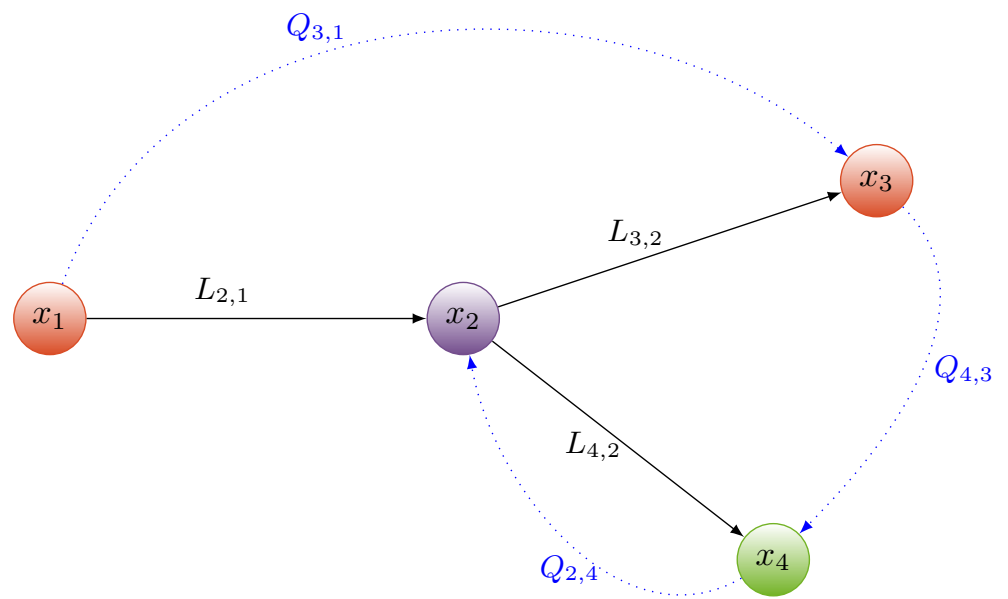

Figure 1: A 3 colors directed coupled network, composed with 4 nodes, exhibiting 3 different internal dynamics, corresponding to 3 different parameter values in system $\dot{x}=f(t, x, \lambda)$.

The set $\mathscr{E}_{L}$ of linear edges is $\{(1,2),(2,3),(2,4)\}$. Thus

$$
L=\left(\begin{array}{cccc}
-L_{2,1} & 0 & 0 & 0 \\
L_{2,1} & -L_{3,2}-L_{4,2} & 0 & 0 \\
0 & L_{3,2} & 0 & 0 \\
0 & L_{4,2} & 0 & 0
\end{array}\right)
$$

The set $\mathscr{E}_{Q}$ of quadratic edges is $\{(1,3),(3,4),(4,2)\}$. Thus

$$
Q(X)=\left(\begin{array}{cccc}
0 & 0 & -Q_{3,1} \kappa\left(x_{1}, x_{3}\right) & 0 \\
0 & 0 & 0 & Q_{2,4} \kappa\left(x_{4}, x_{2}\right) \\
Q_{3,1} \kappa\left(x_{1}, x_{3}\right) & 0 & 0 & -Q_{4,3} \kappa\left(x_{3}, x_{4}\right) \\
0 & -Q_{2,4} \kappa\left(x_{4}, x_{2}\right) & Q_{4,3} \kappa\left(x_{3}, x_{4}\right) & 0
\end{array}\right) .
$$


We consider three distinct parameter values $\lambda_{1}, \lambda_{2}, \lambda_{3}$, and the coupled network given by

$$
\left\{\begin{array}{l}
\dot{x}_{1}=f\left(t, x_{1}, \lambda_{1}\right)-L_{2,1} \mathcal{H} x_{1}-Q_{3,1} \kappa\left(x_{1}, x_{3}\right) \\
\dot{x}_{2}=f\left(t, x_{2}, \lambda_{2}\right)+L_{2,1} \mathcal{H} x_{1}-\left(L_{3,2}+L_{4,2}\right) \mathcal{H} x_{2}+Q_{2,4} \kappa\left(x_{4}, x_{2}\right) \\
\dot{x}_{3}=f\left(t, x_{3}, \lambda_{1}\right)+L_{3,2} \mathcal{H} x_{2}+Q_{3,1} \kappa\left(x_{1}, x_{3}\right)-Q_{4,3} \kappa\left(x_{3}, x_{4}\right) \\
\dot{x}_{4}=f\left(t, x_{4}, \lambda_{3}\right)+L_{4,2} \mathcal{H} x_{2}+Q_{4,3} \kappa\left(x_{3}, x_{4}\right)-Q_{2,4} \kappa\left(x_{4}, x_{2}\right) .
\end{array}\right.
$$

Let $\left(x_{i}^{(1)}, x_{i}^{(2)}\right)$ denote the components of $x_{i} \in \mathbb{R}^{2}, i \in\{1, \ldots, N\}$. If the first component of $x_{i}$ is coupled, but not the second one, we set:

$$
\mathcal{H}=\left(\begin{array}{ll}
1 & 0 \\
0 & 0
\end{array}\right),
$$

thus $\mathcal{H} x_{i}=\left(x_{i}^{(1)}, 0\right)^{T}$.

The following definition is classical for identical synchronization. For $X_{0}$ in $\Omega$ and $t_{0} \in \mathbb{R}$, we denote by $X\left(t, t_{0}, X_{0}\right) \in \mathbb{R}^{n \times N}$ the orbit of system (2) passing through $X_{0}$ at $t_{0}$, and $x_{i}\left(t, t_{0}, X_{0}\right) \in \mathbb{R}^{n}$ the projection of $X\left(t, t_{0}, X_{0}\right)$ corresponding to the state of node $i$ for any $i \in\{1, \ldots, N\}$.

Definition 1. Two nodes $i$ and $j$ of a coupled network (2) are said to identically synchronize if:

$$
\lim _{t \rightarrow+\infty}\left\|x_{i}\left(t, t_{0}, X_{0}\right)-x_{j}\left(t, t_{0}, X_{0}\right)\right\|_{\mathbb{R}^{n}}=0,
$$

for any initial condition $X_{0} \in \Omega$. We say that the whole coupled network (2) synchronizes if any pair of nodes identically synchronizes.

The main question we are interested in, is to determine the global dynamic of the network. In particular, we would like to know if one node can drive the rest of the network to its internal dynamic, and to analyze the effect of the topology on the bifurcation identified in the dynamical system (1).

\subsection{PCR system}

The PCR system is a model for human behaviors during catastrophic events, established in 2013 to better understand and predict human reactions facing a brutal disaster [21, 22, 27, 5] . It is given by the following adimensional system of ordinary equations:

$$
\left\{\begin{array}{l}
\dot{r}=\gamma(t) q(1-r)-\left(B_{1}+B_{2}\right) r+F(r, c) r c+G(r, p) r p \\
\dot{c}=B_{1} r+C_{1} p-C_{2} c-F(r, c) r c+H(c, p) c p-\varphi(t) c(1-b) \\
\dot{p}=B_{2} r-C_{1} p+C_{2} c-G(r, p) r p-H(c, p) c p \\
\dot{q}=-\gamma(t) q(1-r) \\
\dot{b}=\varphi(t) c(1-b)
\end{array}\right.
$$

for all $t \geq t_{0}$ with a given $t_{0} \in \mathbb{R}$, together with an initial condition $\left(r_{0}, c_{0}, p_{0}, q_{0}, b_{0}\right) \in\left(\mathbb{R}^{+}\right)^{5}$ that satisfies the property

$$
r_{0}+c_{0}+p_{0}+q_{0}+b_{0}=1,
$$

corresponding to the fact that the population densities that we study have been adimensioned.

The unknown functions $r, c, p, q, b$ are real valued functions, and denote respectively the densities of individuals in reflex, control, panic, daily and back to daily behaviors, among a population concerned with the catastrophe. It is worth noting that the daily behavior group is divided into two subgroups corresponding to the unknowns $q$ and $b$, that respectively correspond to the daily behavior before the beginning of the catastrophe, and the daily behavior after the catastrophe. Of particular interest is the initial condition $(0,0,0,1,0)$, which corresponds to the situation of a 
catastrophic event without any alert to the population, and consequently means that all individuals are in a daily behavior before the disaster. Since the sum of the 5 equations in system (10) is equal to 0 , we can reduce the system as follows:

$$
\left\{\begin{array}{l}
\dot{r}=\gamma(t) q(1-r)-\left(B_{1}+B_{2}\right) r+F(r, c) r c+G(r, p) r p \\
\dot{c}=B_{1} r+C_{1} p-C_{2} c-F(r, c) r c+H(c, p) c p-\varphi(t) c(r+c+p+q) \\
\dot{p}=B_{2} r-C_{1} p+C_{2} c-G(r, p) r p-H(c, p) c p \\
\dot{q}=-\gamma(t) q(1-r) .
\end{array}\right.
$$

The expected asymptotic behavior of the orbits of system (12) corresponds to a return of all individuals to a daily behavior after the catastrophe, that is

$$
\lim _{t \rightarrow+\infty} b(t)=1
$$

or equivalently

$$
\lim _{t \rightarrow+\infty}(r(t)+c(t)+p(t)+q(t))=0 .
$$

The functions $\gamma$ and $\varphi$ model respectively the beginning of the disaster, and the return to a daily behavior. They both satisfy the properties

$$
\gamma(t)>0, \quad \varphi(t)>0, \quad \forall t>t_{0}
$$

and

$$
\gamma(t)=\varphi(t)=1, \quad \forall t \geq t_{1},
$$

for a given $t_{1}>t_{0}$. Property (14) implies that the system (12) is non autonomous only for a finite time. This will be useful in the sequel, especially for the study of the global asymptotic stability of the trivial equilibrium, presented in the section ( $(3)$. The parameters $B_{i}>0, C_{i} \geq 0$, $i \in\{1,2\}$, model the behavioral changes of each individual, while the functions $F, G$ and $H$ model the interaction phenomena that act in parallel.

Remark 1. The values of those parameters are partially chosen by a qualitative approach, in collaboration with geographers. Because of the lack of data, the fine numerical calibration of the model is a work in progress, which implies a narrow working association with secure services.

The next proposition summaries the qualitative results of the mathematical analysis.

Proposition 1. For any initial condition $\left(r_{0}, c_{0}, p_{0}, q_{0}\right) \in\left(\mathbb{R}^{+}\right)^{4}$, the system (12) admits a unique global solution whose components are non negative and bounded. If $C_{1}>0, \mathcal{O}(0,0,0,0)$ is the only equilibrium point, and it is locally asymptotically stable. If $C_{1}=0$, the system presents a persistence of panic behavior, that is:

$$
\lim _{t \rightarrow+\infty} p(t)=\bar{p}>0
$$

for any initial condition $\left(r_{0}, c_{0}, p_{0}, q_{0}\right) \in\left(\mathbb{R}^{+}\right)^{4}$ satisfying $r_{0}+c_{0}+p_{0}+q_{0}>0$.

The complete proof is presented in [5], where the authors show that the bifurcation occurring in the system, when the evolution parameter $C_{1}$ passes through 0 , is a degeneracy case of a saddlenode bifurcation at infinity. They also focus on the inhibition role of quadratic terms in the case of a persistence of panic, and highlight the decisive potential role played by the total population density involved in the disaster, that is $\mathcal{T}=r+c+p+q$, which is considered in order to build a Lyapunov function for the stability analysis. This potential role will appear again in the next section.

\section{$\S 3$ Coupled networks of non identical PCR systems}

\subsection{PCR networks}

In what follows, we will consider a PCR network, that is, a graph whose nodes are coupled with non identical instances of the PCR system, whose state equations can be rewritten

$$
\dot{x}=f\left(t, x, C_{1}\right), \quad t \geq t_{0}, \quad x \in \mathbb{R}^{4}, \quad C_{1} \geq 0,
$$


where $x=(r, c, p, q)^{T}$, and $f\left(t, x, C_{1}\right)$ is defined by:

$$
f\left(t, x, C_{1}\right)=\left(\begin{array}{c}
\gamma(t) q(1-r)-\left(B_{1}+B_{2}\right) r+F(r, c) r c+G(r, p) r p \\
B_{1} r+C_{1} p-C_{2} c-F(r, c) r c+H(c, p) c p-\varphi(t) c(r+c+p+q) \\
B_{2} r-C_{1} p+C_{2} c-G(r, p) r p-H(c, p) c p \\
-\gamma(t) q(1-r)
\end{array}\right) .
$$

Definition 2. The nodes which are coupled with an instance of system (12) where $C_{1}=0$ will be called panic nodes or nodes of type (1), while those which are coupled with an instance of system (12) where $C_{1}>0$ will be called control nodes or nodes of type (2).

Remark 2. The value of the evolution parameter $C_{1}$ is chosen according to geographical considerations, among them are the nature of the catastrophe, the position of the nodes with respect to the impact zone of it, but also human behavioral aspects. The example of an earthquake in Japan is studied in [26]. Since in Japan, the risk culture is well established, the population is formed to react quickly, thus the causality process from reflex to control is important with respect to the other processes, which implies a positive value for $C_{1}$, even for a node which is closed to the epicenter of the earthquake. At the opposite, a tsunami on the Mediterranean coast [6] presents a non homogeneous structure. Because of the effect of surprise and fear, reactions are more instinctive in the heart of the disaster zone, namely the beach places. But the particular urban landscape of Mediterranean coastal cities, with dense centers, narrow streets, and elevation over the sea level, is known to hide the arrival of such an event, and represents a quality refuge zone. For those reasons, beach places are modeled by panic nodes, and city center zones by control nodes.

Next, we study a network made of $n$ nodes $x_{i}, 1 \leq i \leq n$ of type (1), and $m$ nodes $y_{j}\left(C_{1}^{j}\right)$, $1 \leq j \leq m$ of type (2), with $C_{1}^{j}>0,1 \leq j \leq m$. The whole network system reads

$$
\dot{X}=\Phi(t, X, C)+L \tilde{X}+\mathcal{Q}(X),
$$

where the vectors $X, \tilde{X} \in \mathbb{R}^{4(n+m)}$ and $C \in \mathbb{R}^{n+m}$ are defined by

$$
\left\{\begin{array}{l}
X=\left(x_{1}, \ldots, x_{n}, y_{1}, \ldots, y_{m}\right)^{T} \\
\tilde{X}=\left(\mathcal{H} x_{1}, \ldots, \mathcal{H} x_{n}, \mathcal{H} y_{1}, \ldots, \mathcal{H} y_{m}\right)^{T} \\
C=\left(0, \ldots, 0, C_{1}^{1}, \ldots, C_{1}^{m}\right)
\end{array}\right.
$$

and $\Phi$ corresponds to the internal dynamic of each node, and is defined by

$$
\Phi(t, X, C)=\left(f\left(t, x_{1}, 0\right), \ldots, f\left(t, x_{n}, 0\right), f\left(t, y_{1}, C_{1}^{1}\right), \ldots, f\left(t, y_{m}, C_{1}^{m}\right)\right)^{T} .
$$

The matrix $\mathcal{H}$ determines which components are coupled:

$$
\mathcal{H}=\left(\begin{array}{llll}
1 & 0 & 0 & 0 \\
0 & 1 & 0 & 0 \\
0 & 0 & 1 & 0 \\
0 & 0 & 0 & 0
\end{array}\right)
$$

The latter definition of $\mathcal{H}$ means that we consider that only individuals in a catastrophe behavior (reflex, control or panic, but not daily behavior) are concerned with migrations from one node to another. The matrix $L$ contains the coupling terms, and can be written as a block matrix:

$$
L=\left(\begin{array}{ll}
L^{1} & L^{2} \\
L^{3} & L^{4}
\end{array}\right)
$$

where $L^{1}=\left(L_{i, j}^{1}\right), 1 \leq i, j \leq n$, corresponds to the couplings among the nodes of type (1), $L^{2}=\left(L_{i, j}^{2}\right), 1 \leq i \leq n, 1 \leq j \leq m$, and $L^{3}=\left(L_{i, j}^{3}\right), 1 \leq i \leq m, 1 \leq j \leq n$, to the couplings between nodes of type (1) and nodes of type (2), and $L^{4}=\left(L_{i, j}^{4}\right), 1 \leq i, j \leq m$, to the couplings among the nodes of type (2) (see Figure 2).

The quadratic couplings are stored in $\mathcal{Q}(X)=\left(\mathcal{Q}^{1}(X), \mathcal{Q}^{2}(X)\right)$, which is defined by the coefficients of the matrix $Q(X)$ (see (4) and (6)), and the function $\kappa$, given by

$$
\kappa(x, \tilde{x})=\left(x_{1}\left(\tilde{x}_{2}+\tilde{x}_{3}\right), x_{2}\left(\tilde{x}_{1}+\tilde{x}_{3}\right), x_{3}\left(\tilde{x}_{1}+\tilde{x}_{2}\right), 0\right)^{T},
$$

for all $x, \tilde{x} \in \mathbb{R}^{4}$. This expression models the situation when individuals of node $i$ imitate individuals of node $j$ of another behavior group, except those who are in the daily behavior. 

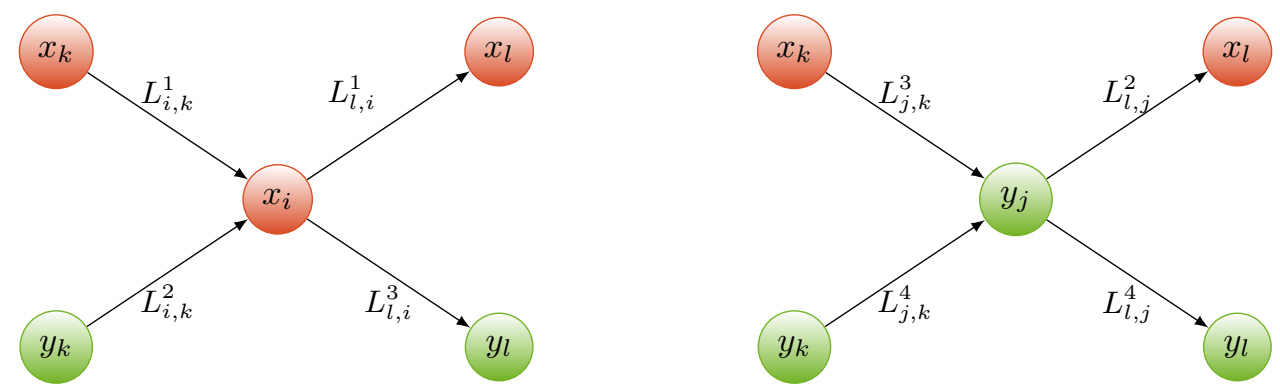

Figure 2: Notations for the linear coupling terms in a PCR network. Red models panic nodes, while green represents control nodes.

Remark 3. It is observed by geographers that the physical displacements are of two types. The first type is the result of a crowd motion in which the behavioral evolution is almost frozen, especially when the time of displacement is short. We have chosen to model this type of displacements by linear couplings. The second type occurs when a communication takes place between individuals standing on different places. This communication corresponds to an interaction which can take hold through a vocal message, a mobile call, or just arms signs in the case of two nodes which are separated by a tiny distance. Thus, we have chosen to model the second type of displacements by quadratic couplings.

Let us consider the concrete example of an earthquake in a low density zone in Japan. Let $\left(r_{1}, c_{1}, p_{1}\right)$ and $\left(r_{2}, c_{2}, p_{2}\right)$ denote the reflex, control and panic behaviors on two nodes 1 and 2 respectively. We suppose that the node 1 is located at the epicenter of the catastrophe, and thus can present a persistence of panic in absence of coupling, whereas the node 2 corresponds to a refuge zone which favors the return to a daily behavior. In that case, the equations of $\dot{p}_{1}, \dot{p}_{2}, \dot{c}_{2}$ read as follows:

$$
\left\{\begin{array}{l}
\dot{p}_{1}=f_{1}\left(r_{1}, c_{1}, p_{1}\right)-\varepsilon_{L} p_{1}-\varepsilon_{Q} p_{1} c_{2} \\
\dot{p}_{2}=f_{2}\left(r_{2}, c_{2}, p_{2}\right)+\varepsilon_{L} p_{1} \\
\dot{c}_{2}=f_{3}\left(r_{2}, c_{2}, p_{2}\right)+\varepsilon_{Q} p_{1} c_{2}
\end{array}\right.
$$

where $f_{1}, f_{2}, f_{3}$ correspond to the internal dynamic in each equation (see (15)). The linear coupling $\pm \varepsilon_{L} p_{1}$ models the displacements of individuals in panic behavior from node 1 towards node 2. During this displacement, no behavioral evolution occurs. In a different manner, the quadratic coupling $\pm \varepsilon_{Q} p_{1} c_{2}$ is the result of an interaction, say a message which is spread by rescue services through loudspeakers, and leads simultaneously to a displacement and a behavioral evolution: individuals in panic near the epicenter are calmed down by the rescue message, thus arrive at the refuge zone in a control behavior.

For clarity, we adopt the coloring representation that associates red to the panic nodes, and green to the control nodes (see Figure 2). The PCR network (16) can be rewritten in an expanded form:

$$
\left\{\begin{array}{c}
\dot{x}_{i}=f\left(t, x_{i}, 0\right)-\left(\sum_{\substack{k=1 \\
k \neq i}}^{n} L_{k, i}^{1}+\sum_{l=1}^{m} L_{l, i}^{3}\right) \mathcal{H} x_{i}+\sum_{\substack{k=1 \\
k \neq i}}^{n} L_{i, k}^{1} \mathcal{H} x_{k}+\sum_{l=1}^{m} L_{i, l}^{2} \mathcal{H} y_{l}+\mathcal{Q}_{i}^{1}(X), \\
\dot{y}_{j}=f\left(t, y_{j}, C_{1}^{j}\right)-\left(\sum_{k=1}^{n} L_{k, j}^{2}+\sum_{\substack{l=1 \\
l \neq j}}^{m} L_{l, j}^{4}\right) \mathcal{H} y_{j}+\sum_{k=1}^{n} L_{j, k}^{3} \mathcal{H} x_{k}+\sum_{\substack{l=1 \\
l \neq j}}^{m} L_{j, l}^{4} \mathcal{H} y_{l}+\mathcal{Q}_{j}^{2}(X),
\end{array}\right.
$$

where $x_{i} \in \mathbb{R}^{4}, 1 \leq i \leq n$ and $y_{j} \in \mathbb{R}^{4}, 1 \leq j \leq m$. In order to distinguish the components of the nodes of type (1) and (2), we introduce the following notation:

$$
\begin{aligned}
& x_{i}=\left(r_{i}^{(1)}, c_{i}^{(1)}, p_{i}^{(1)}, q_{i}^{(1)}\right)^{T}, \quad 1 \leq i \leq n, \\
& y_{j}=\left(r_{j}^{(2)}, c_{j}^{(2)}, p_{j}^{(2)}, q_{j}^{(2)}\right)^{T}, \quad 1 \leq j \leq m .
\end{aligned}
$$


Remark 4. The PCR networks that we study are inspired by some geographical configurations [22], which determine the values of the parameters of each node, according to their spatial position, and the coupling strengths, according to the dimensions of the communication paths. Thus, the geographical metric structure of the zone affected by the catastrophe is implicitly taken into account in the parameters of the network. More precisely, the coupling strength along one edge is inversely proportional to the geographical distance between the two corresponding nodes.

Proposition 2. For any initial condition $X_{0} \in\left(\mathbb{R}^{+}\right)^{4(n+m)}$, there exists $\tau>0$ such that the Cauchy problem defined by (16) and $X\left(t_{0}\right)=X_{0}$ admits a unique solution $X\left(t, t_{0}, X_{0}\right)$ defined on $\left[t_{0}, t_{0}+\tau[\right.$, whose components are non negative.

We skip the proof, since it is very similar to the proof of positiveness of the solution of the PCR system (12), detailed in [5]. In the absence of coupling, the panic nodes $x_{i}, 1 \leq i \leq n$ will exhibit a panic persistence, while the control nodes $y_{j}, 1 \leq j \leq m$ will present a return to daily behavior.

Next, we consider the total population density $\mathcal{T}$ involved in the disaster, defined by:

$$
\mathcal{T}(X)=\sum_{i=1}^{n}\left(r_{i}^{(1)}+c_{i}^{(1)}+p_{i}^{(1)}+q_{i}^{(1)}\right)+\sum_{j=1}^{m}\left(r_{j}^{(2)}+c_{j}^{(2)}+p_{j}^{(2)}+q_{j}^{(2)}\right) .
$$

Proposition 3. The function $\mathcal{T}$ is non negative and decreasing on the interval $\left[t_{0}, t_{0}+\tau[\right.$ along the solution $X\left(t, t_{0}, X_{0}\right)$ of the PCR network starting from an initial condition $X_{0} \in\left(\mathbb{R}^{+}\right)^{4(n+m)}$. Assume that the solution is global, that is $\tau=+\infty$. If $\mathcal{T}$ converges to 0 , then the PCR network (20) synchronizes towards the trivial equilibrium.

Proof. The sum of the $4(n+m)$ equations in $(20)$ makes the coupling terms vanish and leads to

$$
\begin{aligned}
& \dot{\mathcal{T}}\left(X\left(t, t_{0}, X_{0}\right)\right)= \\
& -\varphi(t)\left(\sum_{i=1}^{n} c_{i}^{(1)}(t)\left(r_{i}^{(1)}+c_{i}^{(1)}+p_{i}^{(1)}+q_{i}^{(1)}\right)(t)+\sum_{j=1}^{m} c_{j}^{(2)}(t)\left(r_{j}^{(2)}+c_{j}^{(2)}+p_{j}^{(2)}+q_{j}^{(2)}\right)(t)\right),
\end{aligned}
$$

thus we have $\dot{\mathcal{T}} \leq 0$. Next, the non negativity of the components (see Proposition 2 ) implies that

$$
0 \leq r_{i}^{(1)}(t) \leq \mathcal{T}(t), \quad 0 \leq c_{i}^{(1)}(t) \leq \mathcal{T}(t), \quad 0 \leq p_{i}^{(1)}(t) \leq \mathcal{T}(t), \quad 0 \leq q_{i}^{(1)}(t) \leq \mathcal{T}(t), \quad 1 \leq i \leq n,
$$

and similarly for the components $r_{j}^{(2)}, c_{j}^{(2)}, p_{j}^{(2)}$ and $q_{j}^{(2)}, 1 \leq j \leq m$. Thus, if $\mathcal{T}$ converges to 0 , then necessarily all the components of the nodes of type (1) and (2) converge to 0 , that is the PCR network synchronizes towards the trivial equilibrium.

The total population density $\mathcal{T}$ is also likely not to converge to 0 , but to a positive limit. In that case, the network is susceptible to exhibit a persistence of panic. The complete answer to that question will be given at the end of the section, as a consequence of Theorem 1. Let us consider the energy function

$$
V=\frac{1}{2} \mathcal{T}^{2}
$$

It is non negative, with a non positive orbital derivative by virtue of Proposition 3 , and it enables us to construct an invariant region.

Proposition 4. The compact set $\Omega=\left\{X \in\left(\mathbb{R}^{+}\right)^{4(n+m)}, V(X) \leq 1\right\}$ is a positively invariant region for the flow induced by the PCR network (20).

Proof. Let us consider $X_{0} \in \Omega$. Since $\dot{V}\left(X\left(t, t_{0}, X_{0}\right)\right) \leq 0$, then $V\left(X\left(t, t_{0}, X_{0}\right)\right)$ is decreasing. Therefore, $X\left(t, t_{0}, X_{0}\right)$ remain in $\Omega$ for all future time.

Consequently, the solution of the PCR network starting from $X_{0} \in \Omega$ is global. In the rest of the paper, we study the effect of the coupling in the network.

Definition 3. We will say that the PCR network (16) presents a global return to daily behavior if:

$$
\lim _{t \rightarrow+\infty}\left\|X\left(t, t_{0}, X_{0}\right)\right\|_{\mathbb{R}^{4(n+m)}}=0
$$

for any $X_{0} \in \Omega$. We will say that a panic node $(x)$ is evacuated towards a control node $(y)$ by a linear chain, if there exists an oriented path in the subsequent graph made of linear edges that connects $(x)$ to $(y)$. 
Two nodes converging to the trivial equilibrium $\mathcal{O} \in \mathbb{R}^{4}$ for any initial condition obviously synchronize in the sense of definition (1), while two nodes exhibiting a panic persistence are susceptible not to synchronize. The global return of a PCR network to daily behavior, which is the expected dynamic, is a sufficient condition for synchronization, since the solution is attracted to the trivial equilibrium $\mathcal{O} \in \mathbb{R}^{4(n+m)}$. When a panic node $(x)$ is evacuated towards a control node $(y)$, there may be multiple paths connecting $(x)$ to $(y)$. The length of one path is the number of edges along it (see Figure 6).

\subsection{Patterns emerging from two-nodes configurations}

In this section, we study two-nodes PCR networks (see Figure 3), considering a non symmetric coupling, with a linear or quadratic form, and show which patterns emerge from those basic configurations.

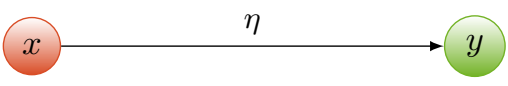

(a)

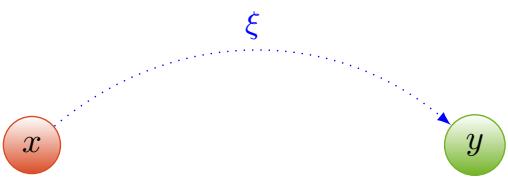

(c)

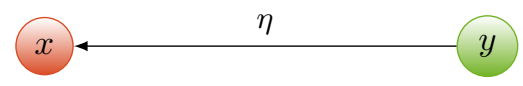

(b)

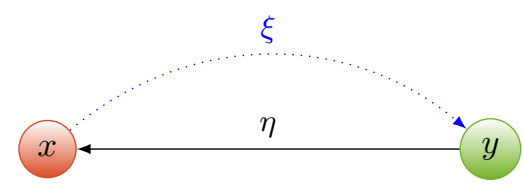

(d)

Figure 3: Two-nodes PCR networks. (a) Linear connection from a panic node towards a control node. (b) Linear connection from a control node towards a panic node. (c) Quadratic connection from a panic node towards a control node. (d) Superposition of quadratic and linear connections of opposite directions between a panic node and a control node.

We first consider a two-nodes PCR network with a panic node $(x)$ connected to a control node $(y)$ in a linear form (see Figure 3(a)). Such a network is given by the following system:

$$
\left\{\begin{array}{l}
\dot{x}=f(t, x, 0)-\eta \mathcal{H} x \\
\dot{y}=f\left(t, y, C_{1}\right)+\eta \mathcal{H} x
\end{array}\right.
$$

where $x=\left(r_{1}, c_{1}, p_{1}, q_{1}\right)^{T}, y=\left(r_{2}, c_{2}, p_{2}, q_{2}\right)^{T}, C_{1}>0, \mathcal{H}$ is defined by (17), and $\eta>0$ corresponds to the coupling strength.

Proposition 5. The system (25) admits a unique equilibrium $\mathcal{O} \in \mathbb{R}^{8}$. Furthermore, it is locally asymptotically stable.

Proof. We first look for the equilibrium points of node $(x)$, which can be seen as a perturbation of a PCR system. To that aim, we solve:

$$
\left\{\begin{array}{l}
\gamma(t) q_{1}\left(1-r_{1}\right)-\left(B_{1}+B_{2}\right) r_{1}+F\left(r_{1}, c_{1}\right) r_{1} c_{1}+G\left(r_{1}, p_{1}\right) r_{1} p_{1}-\eta r_{1}=0 \\
B_{1} r_{1}-C_{2} c_{1}-F\left(r_{1}, c_{1}\right) r_{1} c_{1}+H\left(c_{1}, p_{1}\right) c_{1} p_{1}-\varphi(t) c_{1}\left(r_{1}+c_{1}+p_{1}+q_{1}\right)-\eta c_{1}=0 \\
B_{2} r_{1}+C_{2} c_{1}-G\left(r_{1}, p_{1}\right) r_{1} p_{1}-H\left(c_{1}, p_{1}\right) c_{1} p_{1}-\eta p_{1}=0 \\
-\gamma(t) q_{1}\left(1-r_{1}\right)=0 .
\end{array}\right.
$$

The sum of the 4 equations leads to:

$$
-\varphi(t) c_{1}\left(r_{1}+c_{1}+p_{1}+q_{1}\right)-\eta\left(r_{1}+c_{1}+p_{1}\right)=0
$$

thus $r_{1}=c_{1}=p_{1}=q_{1}=0$, since the components of the solution are non negative. We then write the 4 equations of node $(x)$ as:

$$
\dot{x}=M x+N(t) x+\epsilon(t, x),
$$


where $M$ and $N(t)$ are two matrices of order 4 defined by:

$$
M=\left(\begin{array}{cccc}
-B_{1}-B_{2}-\eta & 0 & 0 & 1 \\
B_{1} & -C_{2}-\eta & 0 & 0 \\
B_{2} & C_{2} & -\eta & 0 \\
0 & 0 & 0 & -1
\end{array}\right), \quad N(t)=\left(\begin{array}{cccc}
0 & 0 & 0 & \gamma(t)-1 \\
0 & 0 & 0 & 0 \\
0 & 0 & 0 & 0 \\
0 & 0 & 0 & -\gamma(t)+1
\end{array}\right),
$$

and $\epsilon$ contains non linear terms:

$$
\epsilon(t, x)=\left(\begin{array}{c}
-\gamma(t) q_{1} r_{1}+F\left(r_{1}, c_{1}\right) r_{1} c_{1}+G\left(r_{1}, p_{1}\right) \\
-F\left(r_{1}, c_{1}\right) r_{1} c_{1}+H\left(c_{1}, p_{1}\right) c_{1} p_{1}-\varphi(t) c_{1}\left(r_{1}+c_{1}+p_{1}+q_{1}\right) \\
-G\left(r_{1}, p_{1}\right) r_{1} p_{1}-H\left(c_{1}, p_{1}\right) c_{1} p_{1} \\
\gamma(t) q_{1} r_{1}
\end{array}\right) .
$$

The eigenvalues of $M$ are given by:

$$
-B_{1}-B_{2}-\eta, \quad-C_{2}-\eta, \quad-\eta, \quad-1,
$$

thus they are negative, since $\eta>0$. Furthermore, some basic algebraic computations, and property (14), lead to:

$$
\lim _{t \rightarrow+\infty}\|N(t)\|=0, \quad \lim _{\|x\| \rightarrow 0} \frac{\|\epsilon(t, x)\|}{\|x\|}=0, \text { uniformly in } t .
$$

The smoothness of functions $\gamma, \varphi, F, G$ and $H$ implies that of $\epsilon$, which is consequently locally Lipschitz in its second argument. Thus the Poincaré-Lyapunov theorem (see [28] for instance) applies and guarantees that the equilibrium $(0,0,0,0)$ is locally asymptotically stable. Finally, we look for the equilibrium points of node $(y)$, which are given by:

$$
\left\{\begin{array}{l}
\gamma(t) q_{2}\left(1-r_{2}\right)-\left(B_{1}+B_{2}\right) r_{2}+F\left(r_{2}, c_{2}\right) r_{2} c_{2}+G\left(r_{2}, p_{2}\right) r_{2} p_{2}+\eta r_{1}=0 \\
B_{1} r_{2}+C_{1} p_{2}-C_{2} c_{2}-F\left(r_{2}, c_{2}\right) r_{2} c_{2}+H\left(c_{2}, p_{2}\right) c_{2} p_{2}-\varphi(t) c_{2}\left(r_{2}+c_{2}+p_{2}+q_{2}\right)+\eta c_{1}=0 \\
B_{2} r_{2}-C_{1} p_{2}+C_{2} c_{2}-G\left(r_{2}, p_{2}\right) r_{2} p_{2}-H\left(c_{2}, p_{2}\right) c_{2} p_{2}+\eta p_{1}=0 \\
-\gamma(t) q_{2}\left(1-r_{2}\right)=0 .
\end{array}\right.
$$

Since $\left(r_{1}, c_{1}, p_{1}, q_{1}\right)=(0,0,0,0)$ is the only equilibrium point of node $(x)$, we obtain the equations corresponding to the equilibrium points of a PCR system (12) with $C_{1}>0$. Thus $\left(r_{2}, c_{2}, p_{2}, q_{2}\right)=(0,0,0,0)$, and the only equilibrium point for the two-nodes network $(25)$ is $0 \in \mathbb{R}^{8}$. Its stability follows from the stability of the equilibrium point $(0,0,0,0)$ of node $(x)$, combined with proposition (1).

Remark 5. This first proposition means that an evacuation of individuals in panic behavior from a panic node towards a control node, brings the whole network to a global return to daily behavior. In other words, the linear coupling affects the bifurcation on the panic node $(x)$, and makes the panic persistence vanish. Furthermore, it will appear in the next paragraph, as a consequence of the general case, that the trivial equilibrium is actually asymptotically globally stable. Thus the two nodes synchronize, and the control node drives the panic node to its dynamic.

We know from theory of ordinary differential equations [9], that any solution $x(t)$ of the first equation in system (25) satisfies the property:

$$
\limsup _{t \rightarrow+\infty} \frac{\ln \|x(t)\|}{t} \leq \max _{1 \leq k \leq 4}\left(\lambda_{k}\right),
$$

where $\lambda_{k}$ denote the eigenvalues of matrix $M$. The latter proof shows that if $\eta<1$, then:

$$
\limsup _{t \rightarrow+\infty} \frac{\ln \|x(t)\|}{t} \leq-\eta,
$$

thus an exponential acceleration of the convergence of node $(x)$ towards the trivial equilibrium under an increase of the coupling strength $\eta$ (see Figure 4).

We then look ahead to the inverse situation, when a control node $(y)$ is connected towards a panic node $(x)$ (see Figure 3(b)):

$$
\left\{\begin{array}{l}
\dot{x}=f(t, x, 0)+\eta \mathcal{H} y \\
\dot{y}=f\left(t, y, C_{1}\right)-\eta \mathcal{H} y
\end{array}\right.
$$




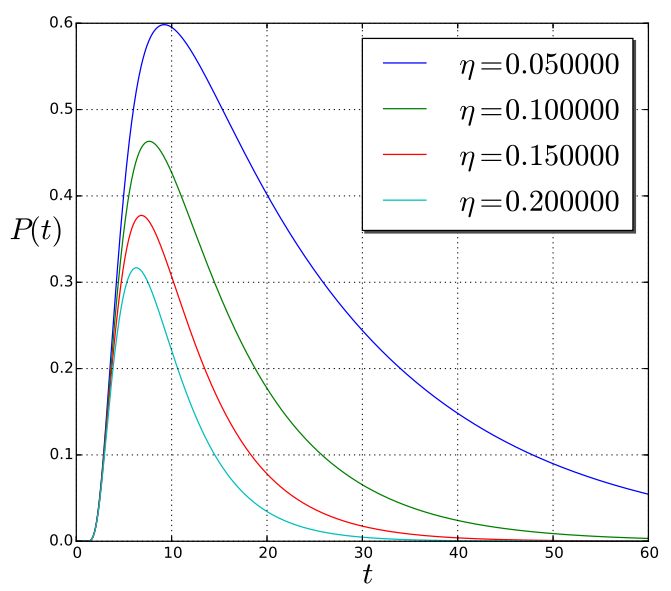

Figure 4: An increase of the coupling strength $\eta$ accelerates the convergence of the total population density $P(t)$ of individuals in panic behavior towards the trivial equilibrium.

Proposition 6. System (26) exhibits a persistence of panic on node $(x)$.

Proof. We begin with the research of the equilibrium points of node $(y)$, which is very similar to the previous proof, and leads to the uniqueness and local asymptotic stability of $\left(r_{2}, c_{2}, p_{2}, q_{2}\right)=$ $(0,0,0,0)$. It follows that the equilibrium points of node $(x)$ correspond to the equilibrium points of a PCR system (12) with $C_{1}=0$, thus the persistence of panic in node $(x)$.

Remark 6. This second proposition suggests that a displacement of individuals of a control node towards a panic node should be avoided, at the risk to worsen the panic persistence level.

Next, we consider a quadratic coupling from a panic node towards a control node (see Figure $3(\mathrm{c}))$ :

$$
\left\{\begin{array}{l}
\dot{x}=f(t, x, 0)-\xi \kappa(x, y) \\
\dot{y}=f\left(t, y, C_{1}\right)+\xi \kappa(x, y),
\end{array}\right.
$$

where the quadratic coupling $\kappa(x, y)$ is defined by (19).

Proposition 7. System (27) presents a persistence of panic on node $(x)$.

We skip the proof, since it is very similar to the latter. Figure 5 shows the shape of the panic component of node $(x)$ for each of the latter situations. Proposition (7) shows that a quadratic coupling is not efficient to empty the panic behavior in the panic nodes, and does not affect the bifurcation in the system.

Finally, we prove that a superposition of a linear and a quadratic couplings (see Figure 3(d)) can inhibit the panic persistence of node $(x)$, by considering the following system:

$$
\left\{\begin{array}{l}
\dot{x}=f(t, x, 0)+\eta \mathcal{H} y-\xi \kappa(x, y) \\
\dot{y}=f\left(t, y, C_{1}\right)-\eta \mathcal{H} y+\xi \kappa(x, y) .
\end{array}\right.
$$

Proposition 8. The solution of system (28) stemming from the initial condition

$$
\left(x_{0}, y_{0}\right)=(0,0,0,1,0,0,0,1)
$$

presents a persistence of panic, which decreases if $\xi$ increases and $\eta$ is sufficiently small.

Proof. The demonstration is based on the Taylor expansion of the solution $X_{\xi}$ of system (28) according to parameter $\xi$. We write:

$$
X_{\xi}=X_{0}+\xi X_{1}+\xi^{2} X_{2}+\ldots,
$$


where the first term $X_{0}$ corresponds to the solution of system (28) with $\xi=0$, and the dots indicate terms of order higher than 2 in $\xi$. After some basic but tedious computations, we obtain:

$$
\left(r_{1}^{(1)}\right)^{(4)}(0)=-3\left(B_{1}+B_{2}\right), \quad\left(c_{1}^{(1)}\right)^{(4)}(0)=-3 B_{1}, \quad\left(p_{1}^{(1)}\right)^{(4)}(0)=-3 B_{2},
$$

while the previous derivatives evaluated in 0 are null. This guarantees the negativeness of the components $r_{1}^{(1)}, c_{1}^{(1)}$ and $p_{1}^{(1)}$ of node (1) on a small interval $\left[t_{0}, t_{0}+\tau\right.$ [. Finally, we prove that $\tau=+\infty$ for $\eta$ sufficiently small, by a similar reasoning as in the proof of the positiveness of the solution of the PCR system (12).

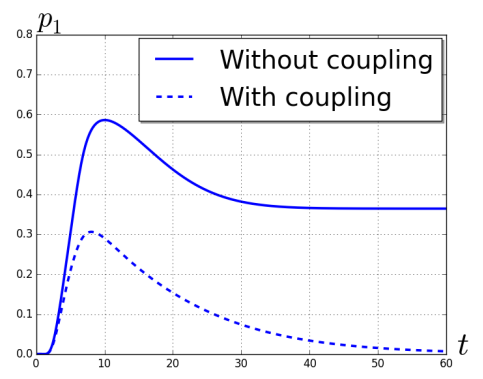

(a)

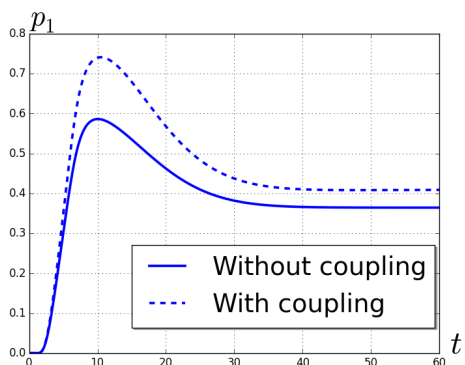

(b)

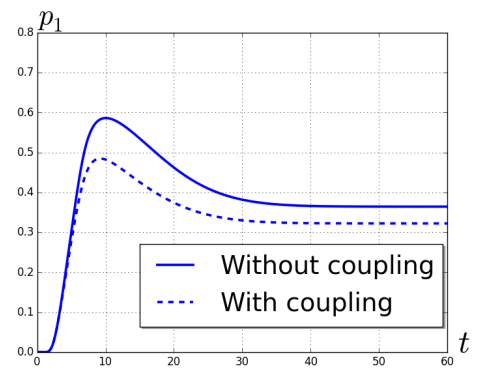

(c)

Figure 5: Panic component of the panic node in a two-nodes PCR network. (a) Linear coupling from panic node to control node: the coupling makes the panic persistence vanish. (b) Linear coupling from control node to panic node: the coupling exacerbates the panic persistence. (c) Quadratic coupling from panic node to control node: the panic persistence survives, but is inhibited.

\subsection{Condition for a global return to normal behavior and global stability of the trivial equilibrium}

In this section, we present and prove our main result, which gives a necessary and sufficient condition for the synchronization of a PCR network. We next analyze the global stability of the trivial equilibrium. To that aim, we introduce, for each $k \geq 1$, the set $\mathcal{P}_{k}$ of all panic nodes which are connected to at least one control node, by an oriented chain of linear edges of length $k$, and not by a shorter chain (see Figure 6). Thus $\mathcal{P}_{k} \cap \mathcal{P}_{l}=\varnothing$ if $k \neq l$. We recall that a panic node can be evacuated towards a control node by multiple paths (see Definition 3), and that the length of such a path is equal to the number of edges along it. A panic node is also likely to be isolated, which means the opposite of being evacuated.

Theorem 1. The PCR network (20) admits a unique equilibrium, which is the trivial equilibrium $\mathcal{O} \in \mathbb{R}^{4(n+m)}$, if and only if every panic node is evacuated by an oriented chain made of linear edges, towards one control node. In that case, the trivial equilibrium is locally stable.

Proof. The proof is divided into three steps. First, we look for the equilibrium points in the system (20), and show that the only components which are not obviously null are the panic components of the panic nodes. Hence, all the quadratic coupling terms vanish in the subsequent equilibrium 


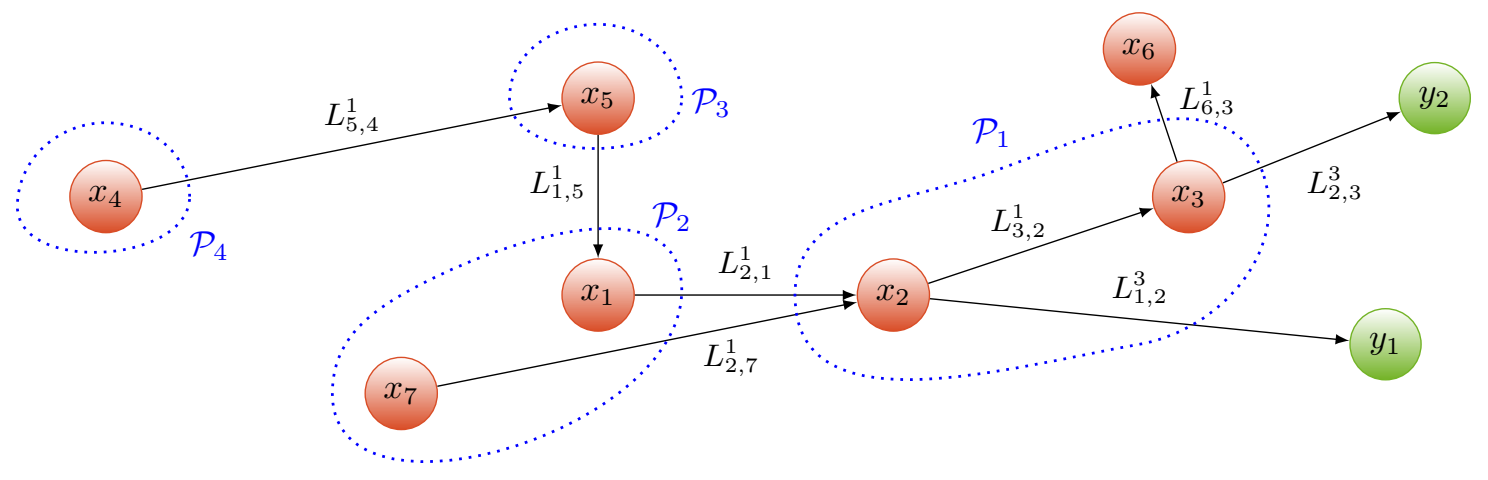

Figure 6: PCR network with different subsets of panic nodes corresponding to the possible evacuation paths towards control nodes. The nodes $x_{2}$ and $x_{3}$ belong to the subset $\mathcal{P}_{1}$ since they are evacuated to one control node by a chain of length 1 . The panic node $x_{6}$ does not belong to any subset $\mathcal{P}_{k}$.

equations. In the second step, we examine if each panic node is evacuated towards a control node by a chain made of linear edges, using an induction reasoning. Finally, we consider a Lyapunov function in order to analyze the local stability of the trivial equilibrium (the global stability will be studied in the next theorem).

First step. In order to find equilibrium points, we solve the following system:

$$
\left\{\begin{array}{l}
f\left(t, x_{i}, 0\right)-\left(\sum_{\substack{k=1 \\
k \neq i}}^{n} L_{k, i}^{1}+\sum_{l=1}^{m} L_{l, i}^{3}\right) \mathcal{H} x_{i}+\sum_{\substack{k=1 \\
k \neq i}}^{n} L_{i, k}^{1} \mathcal{H} x_{k}+\sum_{l=1}^{m} L_{i, l}^{2} \mathcal{H} y_{l}+\mathcal{Q}_{i}^{1}(X)=0 \\
f\left(t, y_{j}, C_{1}^{j}\right)-\left(\sum_{k=1}^{n} L_{k, j}^{2}+\sum_{\substack{l=1 \\
l \neq j}}^{m} L_{l, j}^{4}\right) \mathcal{H} y_{j}+\sum_{k=1}^{n} L_{j, k}^{3} \mathcal{H} x_{k}+\sum_{\substack{l=1 \\
l \neq j}}^{m} L_{j, l}^{4} \mathcal{H} y_{l}+\mathcal{Q}_{j}^{2}(X)=0,
\end{array}\right.
$$

for all $t \geq t_{0}, 1 \leq i \leq n$ and $1 \leq j \leq m$.

The sum of the $4(n+m)$ equations in (29) leads to:

$$
-\varphi(t)\left(\sum_{i=1}^{n} c_{i}^{(1)}\left(r_{i}^{(1)}+c_{i}^{(1)}+p_{i}^{(1)}+q_{i}^{(1)}\right)+\sum_{j=1}^{m} c_{j}^{(2)}\left(r_{j}^{(2)}+c_{j}^{(2)}+p_{j}^{(2)}+q_{j}^{(2)}\right)\right)=0 .
$$

Since $\varphi(t)>0$ for all $t>t_{0}$, and the components of the solution are non negative, we obtain:

$$
c_{i}^{(1)}=0, \quad 1 \leq i \leq n, \quad c_{j}^{(2)}=0, \quad 1 \leq j \leq m .
$$

Next, considering the second components equations, we obtain for nodes of type (1):

$$
B_{1} r_{i}^{(1)}=0, \quad 1 \leq i \leq n,
$$

and for nodes of type (2):

This leads to

$$
B_{1} r_{j}^{(2)}+C_{1}^{j} p_{j}^{(2)}=0, \quad 1 \leq j \leq m .
$$

$$
r_{i}^{(1)}=0, \quad 1 \leq i \leq n, \quad r_{j}^{(2)}=0, \quad 1 \leq j \leq m,
$$

since we have $B_{1}>0$, and similarly:

$$
p_{j}^{(2)}=0, \quad 1 \leq j \leq m,
$$

since $C_{1}^{j}>0$ for all $j \in\{1, \ldots, m\}$. Moreover, we recall that the components $q_{i}^{(1)}, 1 \leq i \leq n$ and $q_{j}^{(2)}, 1 \leq j \leq m$ are not coupled (see (17)). The corresponding equilibrium equations give:

$$
q_{i}^{(1)}=0, \quad 1 \leq i \leq n, \quad q_{j}^{(2)}=0, \quad 1 \leq j \leq m .
$$


Second step. We examine if each panic node is evacuated towards a control node by a chain made of linear edges.

Considering that $p_{j}^{(2)}=0$ for all $j \in\{1, \ldots, m\}$, we obtain for the panic components of the panic nodes:

$$
-\left(\sum_{\substack{k=1 \\ k \neq i}}^{n} L_{k, i}^{1}+\sum_{l=1}^{m} L_{l, i}^{3}\right) p_{i}^{(1)}+\sum_{\substack{k=1 \\ k \neq i}}^{n} L_{i, k}^{1} p_{k}^{(1)}=0,
$$

for all $i \in\{1, \ldots, n\}$. The sum of those $n$ equations gives:

$$
\left(\sum_{l=1}^{m} L_{l, 1}^{3}\right) p_{1}^{(1)}+\left(\sum_{l=1}^{m} L_{l, 2}^{3}\right) p_{2}^{(1)}+\cdots+\left(\sum_{l=1}^{m} L_{l, n}^{3}\right) p_{n}^{(1)}=0,
$$

which can be rewritten:

$$
\lambda_{1} p_{1}^{(1)}+\lambda_{2} p_{2}^{(1)}+\cdots+\lambda_{n} p_{n}^{(1)}=0
$$

where the coefficient $\lambda_{i}=\sum_{l=1}^{m} L_{l, i}^{3}, 1 \leq i \leq n$, corresponds to the possible connections from the panic node $x_{i}$ towards the control nodes $y_{l}, 1 \leq l \leq m$.

First case. Assume that $\mathcal{P}_{1}=\left\{x_{1}, \ldots, x_{n}\right\}$. Then $\lambda_{i}>0$ for $i \in\{1, \ldots, n\}$, which means that all panic nodes are directly connected to at least one control node by a linear oriented edge. We obtain:

$$
p_{i}^{(1)}=0, \forall i \in\{1, \ldots, n\},
$$

which guarantees that $\mathcal{O} \in \mathbb{R}^{4(n+m)}$ is the only equilibrium for the PCR network (20). $\mathcal{T}$ Second case. Assume that $\mathcal{P}_{1}=\varnothing$. Consequently, $\mathcal{P}_{k}=\varnothing$ for all $k \geq 1$, because a panic node which is evacuated by a chain of length $k$, is necessarily connected to a panic node which is evacuated by a chain of length $k-1$. Hence, $\lambda_{i}=0$ for all $i \in\{1, \ldots, n\}$, and $L_{l, j}^{3}=0$ for all $l \in\{1, \ldots, m\}$, $j \in\{1, \ldots, n\}$. Hence, system (30) becomes:

$$
-\left(\sum_{\substack{k=1 \\ k \neq i}}^{n} L_{k, i}^{1}\right) p_{i}^{(1)}+\sum_{\substack{k=1 \\ k \neq i}}^{n} L_{i, k}^{1} p_{k}^{(1)}=0
$$

for all $i \in\{1, \ldots, n\}$, which is a linear system of rank at most $n-1$, and admits non trivial solutions, thus an infinite number of equilibrium points of the form

$$
\bar{X}=\left(\left(0,0, \bar{p}_{i}, 0\right)_{1 \leq i \leq n},(0,0,0,0)_{1 \leq j \leq m}\right),
$$

with arbitrary $\bar{p}_{i}>0$, corresponding to a persistence of panic.

Third case. Assume that $\mathcal{P}_{2} \neq \varnothing$. We can without loss of generality reorder the indexes and write

$$
\lambda_{i}=0, \quad 1 \leq i \leq r_{1}, \quad \lambda_{i}>0, \quad r_{1}+1 \leq i \leq n,
$$

with $1 \leq r_{1} \leq n$, so that $\mathcal{P}_{2} \subset\left\{x_{1}, \ldots, x_{r_{1}}\right\}$ and $\mathcal{P}_{1}=\left\{x_{r_{1}+1}, \ldots, x_{n}\right\}$. Next, we obtain:

$$
\lambda_{r_{1}+1} p_{r_{1}+1}^{(1)}+\cdots+\lambda_{n} p_{n}^{(1)}=0,
$$

which leads to:

$$
p_{i}^{(1)}=0, \forall i \in\left\{r_{1}+1, \ldots, n\right\}
$$

System (30) becomes:

$$
-\left(\sum_{\substack{k=1 \\ k \neq i}}^{n} L_{k, i}^{1}\right) p_{i}^{(1)}+\sum_{\substack{k=1 \\ k \neq i}}^{r_{1}} L_{i, k}^{1} p_{k}^{(1)}=0
$$

for all $i \in\left\{1, \ldots, r_{1}\right\}$. If we sum those $r_{1}$ equations, we get:

$$
\left(\sum_{k=r_{1}+1}^{n} L_{k, 1}^{1}\right) p_{1}^{(1)}+\left(\sum_{k=r_{1}+1}^{n} L_{k, 2}^{1}\right) p_{2}^{(1)}+\cdots+\left(\sum_{k=r_{1}+1}^{n} L_{k, r_{1}}^{1}\right) p_{r_{1}}^{(1)}=0,
$$


which can be rewritten:

$$
\delta_{1} p_{1}^{(1)}+\delta_{2} p_{2}^{(1)}+\cdots+\delta_{r_{1}} p_{r_{1}}^{(1)}=0,
$$

where the coefficient $\delta_{i}=\sum_{k=r_{1}+1}^{n} L_{k, i}^{1}, 1 \leq i \leq r_{1}$ corresponds to the linear connections from the panic nodes $p_{i}^{(1)}, 1 \leq i \leq r_{1}$ (which are not directly evacuated to a control node), towards the panic nodes $p_{i}^{(1)}, r_{1}+1 \leq i \leq n$, which do admit a direct linear connection to at least one control node.

Once again, we distinguish the following three cases:

1. $\mathcal{P}_{2}=\left\{x_{1}, \ldots, x_{r_{1}}\right\}$,

2. $\mathcal{P}_{2}=\varnothing$,

3. $\mathcal{P}_{3} \neq \varnothing$.

An induction reasoning enables us to build a finite sequence of indexes $1 \leq r_{s}<\cdots<r_{2}<$ $r_{1}<n$, such that $\mathcal{P}_{s+1} \subset\left\{x_{1}, \ldots, x_{r_{s}}\right\}$. If $\bigcup_{k=1}^{s+1} \mathcal{P}_{k}=\left\{x_{1}, \ldots, x_{n}\right\}$, then every panic node $p_{i}^{(1)}$ is evacuated towards at least one control node by a linear oriented chain, and simultaneously, we have:

$$
p_{i}^{(1)}=0, \quad 1 \leq i \leq n .
$$

It shows that the only equilibrium in the network corresponds to the global return to daily behavior.

Else, that is, if $\bigcup_{k=1}^{s+1} \mathcal{P}_{k} \varsubsetneqq\left\{x_{1}, \ldots, x_{n}\right\}$, it means that some of the panic nodes are not connected to the control nodes by a linear oriented chain, forming a cluster of panic, admitting equilibrium points of the form

$$
\bar{X}=\left(\left(0,0, \bar{p}_{i}, 0\right)_{1 \leq i \leq r_{s}},(0,0,0,0)_{r_{s}+1 \leq i \leq n+m}\right),
$$

with arbitrary $\bar{p}_{i}>0,1 \leq i \leq r_{s}$.

Third step. To achieve the proof, we analyze the stability of the equilibrium $\mathcal{O} \in \mathbb{R}^{4(n+m)}$. Indeed, this simply follows from the construction of the Lyapunov function $V$ that we have already considered in order to build an invariant region (see Equation (23)), defined by:

$$
V=\frac{1}{2} \mathcal{T}^{2}
$$

where $\mathcal{T}$ denotes the total population density involved in the disaster (see Equation (21)). More precisely, $V$ is semi definite positive. Its orbital derivative is given by

$$
\dot{V}=\mathcal{T} \dot{\mathcal{T}}
$$

But $\dot{\mathcal{T}} \leq 0$ (see Proposition (3)), so $\dot{V}$ is semi definite negative, and this achieves the proof.

This theorem gives a necessary and sufficient condition for the solution of a PCR network to converge to the trivial equilibrium $\mathcal{O} \in \mathbb{R}^{4(n+m)}$, which corresponds to a global return of all individuals to a daily behavior. Its demonstration constructs an oriented chain made of linear edges, from each non isolated panic node to one control node. In practice, those evacuation chains can be determined by running a graph theory algorithm of path finding. Next we analyze the global stability of the trivial equilibrium.

Theorem 2. The trivial equilibrium is globally asymptotically stable if and only if every panic node is evacuated.

Proof. First, assume that the trivial equilibrium is globally asymptotically stable, and that some panic nodes are not evacuated. Then, as pointed in the latter proof, the system (20) admits an infinite number of panic persistence equilibria of the form (36), which are arbitrary close to the trivial equilibrium. Since it is supposed to be globally asymptotically stable, it must be isolated in its basin of attraction, thus a contradiction.

Conversely, assume that every panic node is evacuated. Let us consider the auxiliary system obtained by substituting 1 in place of $\gamma(t)$ and $\varphi(t)$ in the system (16):

$$
\dot{Y}=\hat{\Phi}(t, Y, C)+L \tilde{Y}+\mathcal{Q}(Y)
$$


where the vectors $Y, \tilde{Y} \in \mathbb{R}^{4(n+m)}$ and $C \in \mathbb{R}^{n+m}$ are given by

$$
\left\{\begin{array}{l}
Y=\left(\hat{x}_{1}, \ldots, \hat{x}_{n}, \hat{y}_{1}, \ldots, \hat{y}_{m}\right)^{T} \\
\tilde{Y}=\left(\mathcal{H} \hat{x}_{1}, \ldots, \mathcal{H} \hat{x}_{n}, \mathcal{H} \hat{y}_{1}, \ldots, \mathcal{H} \hat{y}_{m}\right)^{T} \\
C=\left(0, \ldots, 0, C_{1}^{1}, \ldots, C_{1}^{m}\right)
\end{array}\right.
$$

and $\hat{\Phi}$ corresponds to the modified dynamic

$$
\hat{\Phi}(t, Y, C)=\left(\hat{f}\left(t, \hat{x}_{1}, 0\right), \ldots, \hat{f}\left(t, \hat{c}_{n}, 0\right), \hat{f}\left(t, \hat{y}_{1}, C_{1}^{1}\right), \ldots, \hat{f}\left(t, \hat{y}_{m}, C_{1}^{m}\right)\right)^{T}
$$

with

$\hat{f}\left(t, \hat{x}, C_{1}\right)=\left(\begin{array}{c}\hat{q}(1-\hat{r})-\left(B_{1}+B_{2}\right) \hat{r}+F(\hat{r}, \hat{c}) \hat{r} \hat{c}+G(\hat{r}, \hat{p}) \hat{r} \hat{p} \\ B_{1} \hat{r}+C_{1} \hat{p}-C_{2} \hat{c}-F(\hat{r}, \hat{c}) \hat{r} \hat{c}+H(\hat{c}, \hat{p}) \hat{c} \hat{p}-\hat{c}(\hat{r}+\hat{c}+\hat{p}+\hat{q}) \\ B_{2} \hat{r}-C_{1} \hat{p}+C_{2} \hat{c}-G(\hat{r}, \hat{p}) \hat{r} \hat{p}-H(\hat{c}, \hat{p}) \hat{c} \hat{p} \\ -\hat{q}(1-\hat{r})\end{array}\right), \hat{x}=(\hat{r}, \hat{c}, \hat{p}, \hat{q})$,

the rest of the system being unmodified, in particular the couplings terms. Let $V$ be the Lyapunov function defined by (37). It is easily seen that the set $\Omega$ introduced in Proposition 4 is also an invariant region for the modified system (39). Let define $E=\{Y \in \Omega ; \dot{V}(Y)=0\}$. Some basic computations show that $Y \in E$ if and only if $\hat{c}_{i}^{(1)}=\hat{c}_{j}^{(2)}=0$ for all $i \in\{1, \ldots, n\}$ and $j \in\{1, \ldots, m\}$. The La Salle invariance theorem (see $[18,15,14]$ ) guarantees that the orbits of system (39) approach the largest invariant set $M$ included in $E$.

Next, we show that $M$ is not more than the trivial equilibrium. So, let us consider an orbit $Y\left(t, t_{0}, Y_{0}\right)$ of system (39) starting from an initial condition $Y_{0} \in M$ at $t=t_{0}$. Since $M \subset E$, we have $\hat{c}_{i}^{(1)}(t)=\hat{c}_{j}^{(2)}(t)=0$ for all $i \in\{1, \ldots, n\}$ and $j \in\{1, \ldots, m\}$ and for all $t$. The corresponding equations in system (39) become:

$$
\begin{aligned}
& \dot{\hat{c}}_{i}^{(1)}=B_{1} \hat{r}_{i}^{(1)}, \quad 1 \leq i \leq n, \\
& \dot{\hat{\hat{c}}}_{j}^{(2)}=B_{1} \hat{r}_{j}^{(2)}+C_{1} \hat{p}_{j}^{(2)}, \quad 1 \leq j \leq m .
\end{aligned}
$$

Necessarily, we have $\hat{r}_{i}^{(1)}(t)=0$ for all $i \in\{1, \ldots, n\}, t \geq t_{1}$ and $\hat{r}_{j}^{(2)}(t)=\hat{p}_{j}^{(2)}(t)=0$ for all $j \in\{1, \ldots, m\}, t \geq t_{1}$. Similarly, the equations of the reflex behavior component $\hat{r}$ become:

$$
\begin{aligned}
& \dot{\hat{r}}_{i}^{(1)}=\hat{q}_{i}^{(1)}, \quad 1 \leq i \leq n, \\
& \dot{\hat{r}}_{j}^{(2)}=\hat{q}_{j}^{(2)}, \quad 1 \leq j \leq m .
\end{aligned}
$$

which proves that $\hat{q}_{i}^{(1)}(t)=0$ and $\hat{q}_{j}^{(2)}(t)=0$ for all $i, j, t$. Then we consider $\hat{x}_{i} \in \mathcal{P}_{1}$. By assumption, there exists a control node $\hat{y}_{j}$ such that $\hat{x}_{i}$ is connected to $\hat{y}_{j}$. The equation of the panic component of node $\hat{y}_{j}$ becomes:

$$
\dot{\hat{p}}_{j}^{(2)}=\sum_{k=1}^{n} L_{j, k}^{3} \hat{p}_{k}^{(1)} \geq L_{j, i}^{3} \hat{p}_{i}^{(1)}
$$

thus $\hat{p}_{i}^{(1)}(t)=0$ for all $t \geq t_{1}$, since $L_{j, i}^{3}>0$. Analogously, we show that $\hat{p}_{i}^{(1)}(t)=0$ for all $t \geq t_{1}$ and $i \in\{1, \ldots, n\}$, since by assumption every panic node is evacuated.

Finally, let us consider an orbit $X\left(t, t_{0}, X_{0}\right)$ of the original system (16), starting from an initial condition $X_{0} \in \Omega$ at $t=t_{0}$. Because of Proposition (4), we have $X\left(t, t_{0}, X_{0}\right) \in \Omega$ for all $t \geq t_{0}$. Furthermore, property (14) guarantees that $\gamma(t)=\varphi(t)=1$ for all $t \geq t_{1}$, which implies

$$
X\left(t, t_{0}, X_{0}\right)=Y\left(t, t_{1}, X_{1}\right), \quad \forall t \geq t_{1},
$$

where $X_{1}=X\left(t_{1}, t_{0}, X_{0}\right)$. The previous reasoning guarantees that $Y\left(t, t_{1}, X_{1}\right)$ converges to the trivial equilibrium, so the same is for $X\left(t, t_{0}, X_{0}\right)$, and this achieves the proof. 


\section{$\S 4 \quad$ Numerical simulations}

The aim of this section is to illustrate the previous qualitative results by numerical simulations, and to explore the quantitative aspect of the variation of the parameters involved in the PCR networks. The numerical integration has been performed using the scientific library scipy of the python language, on a personal computer equipped with the Debian-GNU/Linux operating system. The networks have been randomly generated by considering random integers for the numbers of linear edges and quadratic edges. The linear edges are randomly associated to the nodes of the networks, whereas the quadratic edges are oriented from the panic nodes towards the control nodes. The graph theory library networkx, which implements a path-finding algorithm [10, 11], is used in order to detect whether the panic nodes are evacuated or not. The functions $\gamma$ and $\varphi$ are given by

$$
\gamma(t)=\zeta(t, 1,3), \quad \varphi(t)=\zeta(t, 1,50),
$$

where $\zeta$ is defined by

$$
\zeta\left(s, s_{0}, s_{1}\right)=\left\{\begin{array}{l}
0 \text { if } s<s_{0} \\
1 \text { if } s>s_{1} \\
\frac{1}{2}-\frac{1}{2} \cos \left(\frac{s-s_{0}}{s_{1}-s_{0}} \pi\right)
\end{array}\right.
$$

and the functions $F, G, H$ are given by

$$
\left\{\begin{array}{l}
F(r, c)=-\alpha_{1} \xi\left(\frac{r}{c+\nu_{0}}\right)+\alpha_{2} \xi\left(\frac{c}{r+\nu_{0}}\right) \\
G(r, p)=-\delta_{1} \xi\left(\frac{r}{p+\nu_{0}}\right)+\delta_{2} \xi\left(\frac{p}{r+\nu_{0}}\right) \\
H(c, p)=-\mu_{1} \xi\left(\frac{c}{p+\nu_{0}}\right)+\mu_{2} \xi\left(\frac{p}{c+\nu_{0}}\right),
\end{array}\right.
$$

where $\nu_{0}=0.01$ and $\xi$ is defined by

$$
\xi(s)=\left\{\begin{array}{l}
1 \text { if } s<0 \\
0 \text { if } s>1 \\
\frac{1}{2}+\frac{1}{2} \cos (\pi s)
\end{array}\right.
$$

The values of the parameters are indicated in Table 1. The values of $B_{1}$ and $B_{2}$ are equal to each other, which means that the evolution processes from reflex to panic, or from reflex to control, are of similar intensity. The value of $C_{1}$ for control nodes is greater than the value of $C_{2}$, which favors the evolution from panic to control. The values of the parameters $\alpha_{i}, \delta_{i}$ and $\mu_{i}, 1 \leq i \leq 2$, involved in the functions $F, G$ and $H$, are lesser than $B_{i}$ and $C_{i}$, which means that the imitation phenomena are dominated by the evolution processes. Along each edge of the network, the linear and quadratic coupling strengths have been fixed at 0.1 . Those numerical values can easily be varied.

The initial condition corresponds, as mentioned previously, to the situation when all individuals are in a daily behavior before the catastrophe, that is

$$
\left\{\begin{array}{lll}
r_{i}^{(1)}=c_{i}^{(1)}=p_{i}^{(1)}=0, & q_{i}^{(1)}=1, & 1 \leq i \leq n, \\
r_{j}^{(2)}=c_{j}^{(2)}=p_{j}^{(2)}=0, & q_{j}^{(2)}=1, & 1 \leq j \leq m .
\end{array}\right.
$$

\subsection{Influence of the topology of the network}

We first consider a particular network made with 15 panic nodes and 15 control nodes, and experiment three different couplings disposals (see Figure 7). 
Table 1: Values of the parameters involved in the PCR network for the numerical simulations. Parameter Numerical value

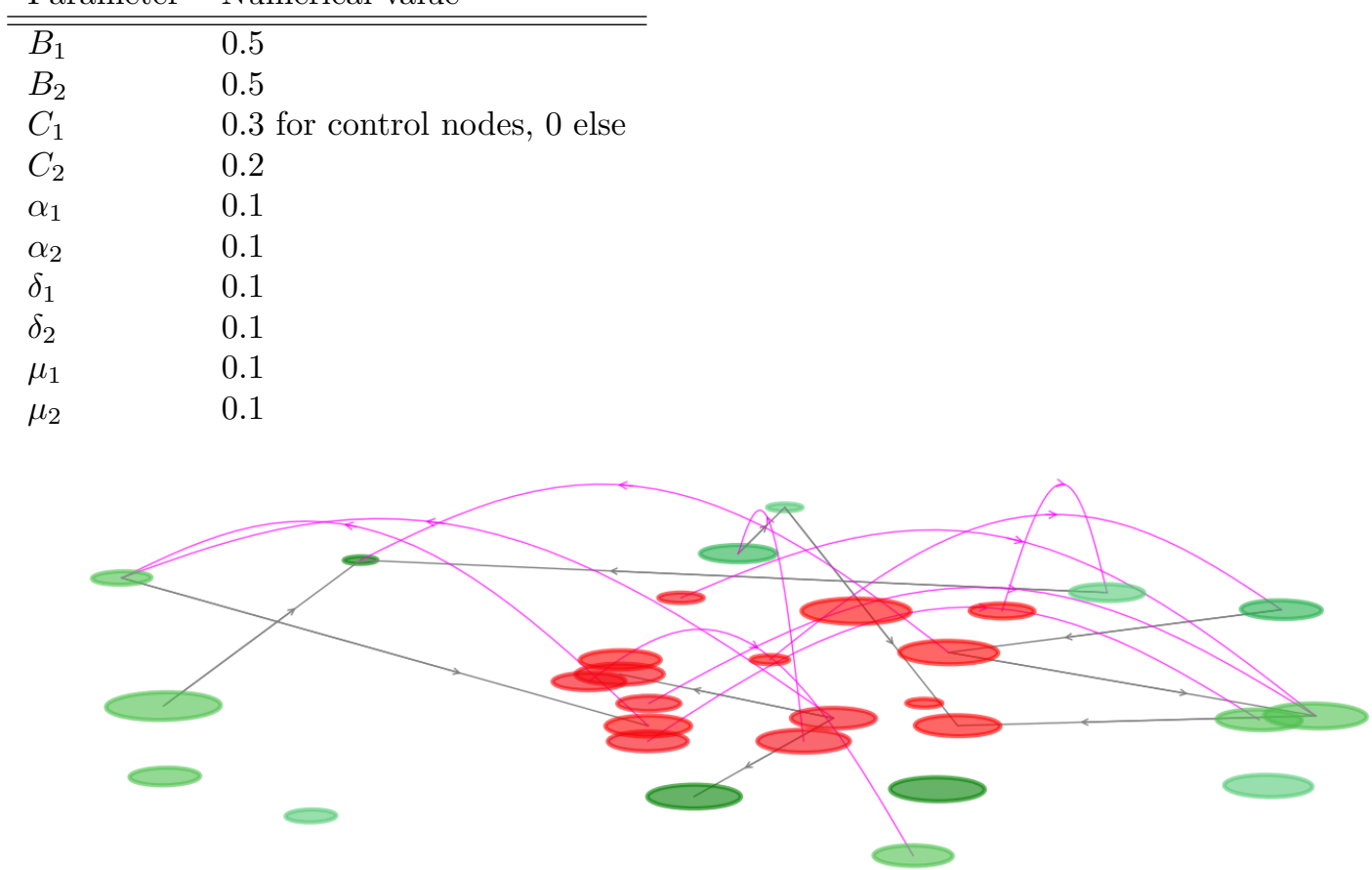

(a)

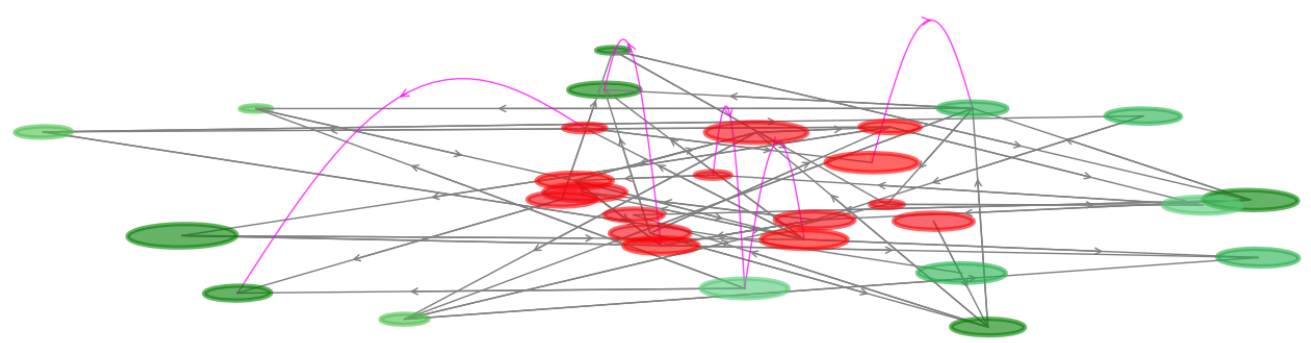

(b)

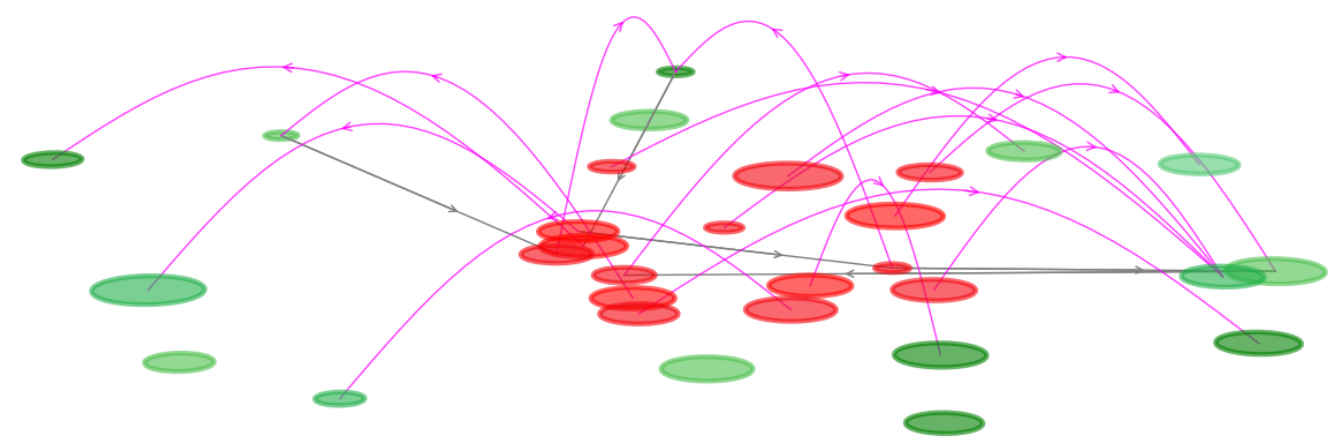

(c)

Figure 7: Three couplings configurations for a particular network. Green models control nodes, while red represents panic nodes. (a) Weakly connected network, without favored orientation between panic and control nodes. (b) Densely connected network, still admitting isolated panic nodes, improved with quadratic couplings from panic nodes towards control nodes. (c) Weakly connected network, with quadratic connections from every panic node towards one control node.

This network is inspired by the geographical background of some epicenter type catastrophe. The position of a node on the affected region influences its nature: when the node is close to 
the heart of the disaster, the panic behavior is predominant. The first configuration is weakly densely connected, with 10 linear couplings and 10 quadratic couplings. It admits 13 isolated panic nodes. Not surprisingly, the network exhibits a high level persistence of panic (see Figures $7(\mathrm{a})$ and $8(\mathrm{a})$ ). The second configuration is densely connected, with 50 linear couplings, but only 5 quadratic couplings. It still admits 3 isolated panic nodes, and thus a lesser persistence of panic (see Figures 7(b) and 8(b)). Finally, the third configuration is made with only 5 linear couplings, and 15 quadratic couplings which are suitably chosen from panic nodes towards control nodes. It admits 13 isolated panic nodes, but the level of panic persistence is attenuated by the quadratic connections, according to Proposition 8.

Remark 7. Those numerical results clearly indicate which choices should be made in order to avoid the persistence of panic behavior in the context of a brutal catastrophic event. Connecting the nodes, which are susceptible to exhibit the highest level of panic, towards other zones which would be a favorable refuge for a return to a daily behavior, is the key of synchronizing the network and avoiding panic persistence. If physical displacements are not possible, one should favor other type interactions to limit the effect of panic. If a cluster of panic nodes is identified, the addition of refuge zones, considered as control nodes, with evacuation paths from the panic nodes, can be sufficient to reach a global return to the daily behavior. At the opposite, a broken connection can have a dramatic consequence, by letting in panic persistence some of the isolated nodes.

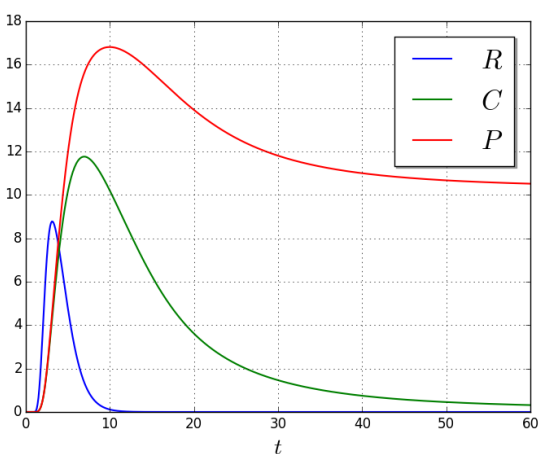

(a)

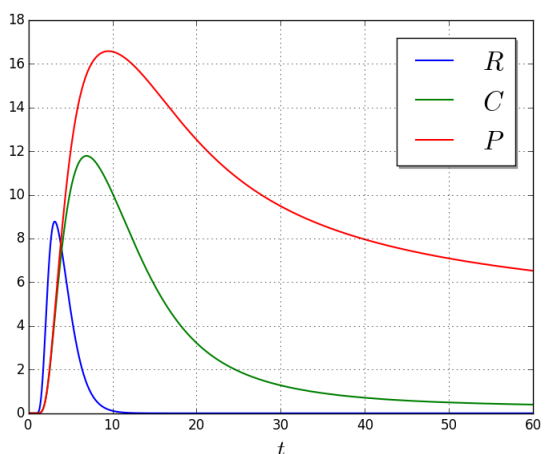

(b)

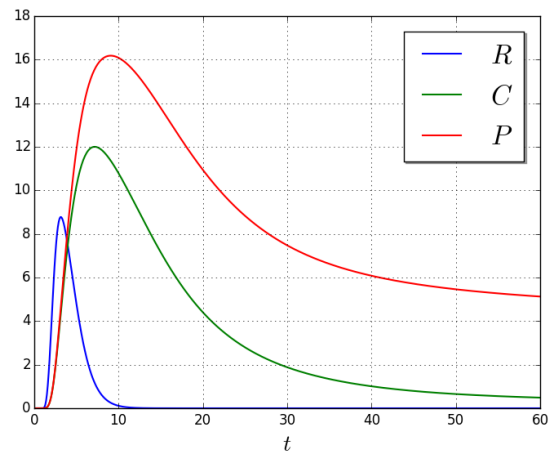

(c)

Figure 8: Numerical results for the three above coupling configurations. (a) The great number of isolated panic nodes accounts for the high level persistence of panic. (b) The low number of isolated panic reduces the panic persistence. (c) The systematic quadratic connection of panic nodes towards control nodes avoids a high level panic persistence.

\subsection{Effect of the length of evacuation chains}

Theorem 1 guarantees that any panic node which is evacuated towards a control node presents a return to daily behavior. The coupling strength can accelerate that convergence, as shown in Figure 4. The length of the evacuation chain is another important parameter. Indeed, let us 
consider a PCR network built with $n$ panic nodes $\left(x_{i}\right), 1 \leq i \leq n$, a unique control node $y$, and linear edges forming a directed chain of length $n$ :

$$
x_{1} \rightarrow x_{2} \rightarrow \cdots \rightarrow x_{n} \rightarrow y \text {. }
$$

Introduce $p_{y}$ the population density in panic behavior in the control node $y$. Figure 9 shows that the convergence of $p_{y}$ towards 0 is as fast as the evacuation chain is short, in conformity with intuition.

Remark 8. This suggests that a preventive organization of high risk zones, with a disposition of refuges which could facilitate the evacuation of individuals of the panic nodes, would accelerate the return to daily behavior among the affected population.

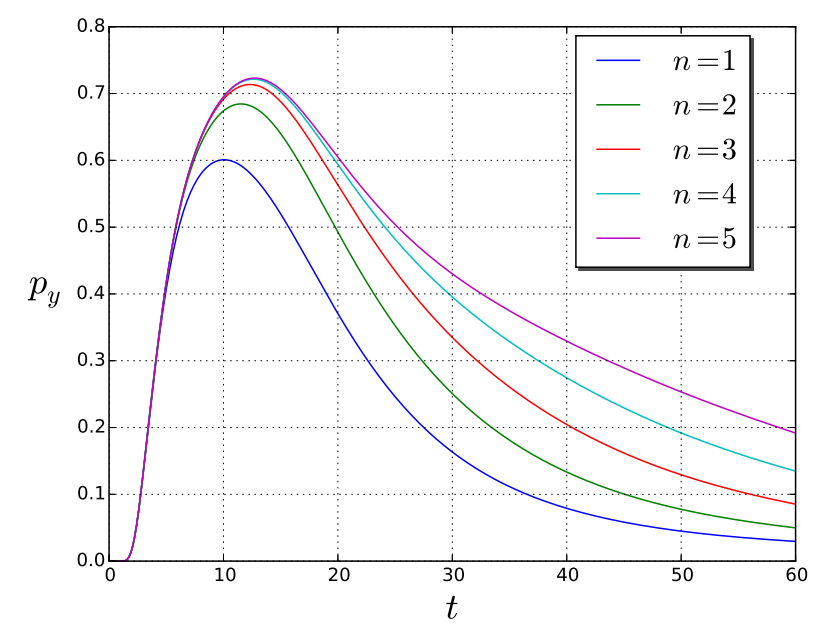

Figure 9: Effect of the length of evacuation chain. The convergence of $p_{y}$ towards 0 is as fast as the evacuation chain is short.

\subsection{Effect of quadratic couplings}

We end our paper with the numerical results of a simulation of 200 randomly generated PCR networks. We compare the solutions of the networks built with only linear couplings, with the solutions of the networks built with the same nodes, the same linear couplings, and additional quadratic couplings chosen from panic nodes towards control nodes. Figure 10 shows the number the relationship between the number of isolated panic nodes and the number of linear edges in the network. More precisely, for each randomly generated network, we compute the number $N_{i}$ of isolated panic nodes by running a path-finding algorithm, and the number $\left|\mathscr{E}_{L}\right|$ of linear edges; each red cross has coordinates $\left(\left|\mathscr{E}_{L}\right|, N_{i}\right)$. Roughly speaking, the number of isolated panic nodes decreases when the number of linear edges increases. However, as mentioned previously, a densely connected network is not an absolute warranty for avoiding panic persistence. A clever disposal of a low number linear edges can be sufficient to evacuate $n$ panic nodes. Theoretically, $n$ linear edges should be sufficient. But the consideration of the geographical relief of the zone affected by the catastrophe can make that disposal become a complex problem.

Remark 9. The shape of the cloud of points in Figure 10 suggests an approximation by an inverse power law of the type $N_{i}=\frac{k_{1}}{\left|\mathscr{E}_{i}\right|^{\nu}}-k_{2}$, with positive constants $k_{1}, k_{2}$ and $\nu$. The emergence of such heuristic laws occurs frequently in the study of complex systems arising in various domains of physics, biology or social sciences, and is linked to the self-organization feature of complex networks (see for instance [20], [13], [1], [7] and the references therein).

Figure 11 illustrates Proposition 8 by comparing the solutions of the PCR networks with or without the effect of quadratic couplings. The blue dots indicate that the density $P_{Q}$ of individuals in panic behavior after a given time $T$, in a PCR network with both linear and quadratic couplings, 


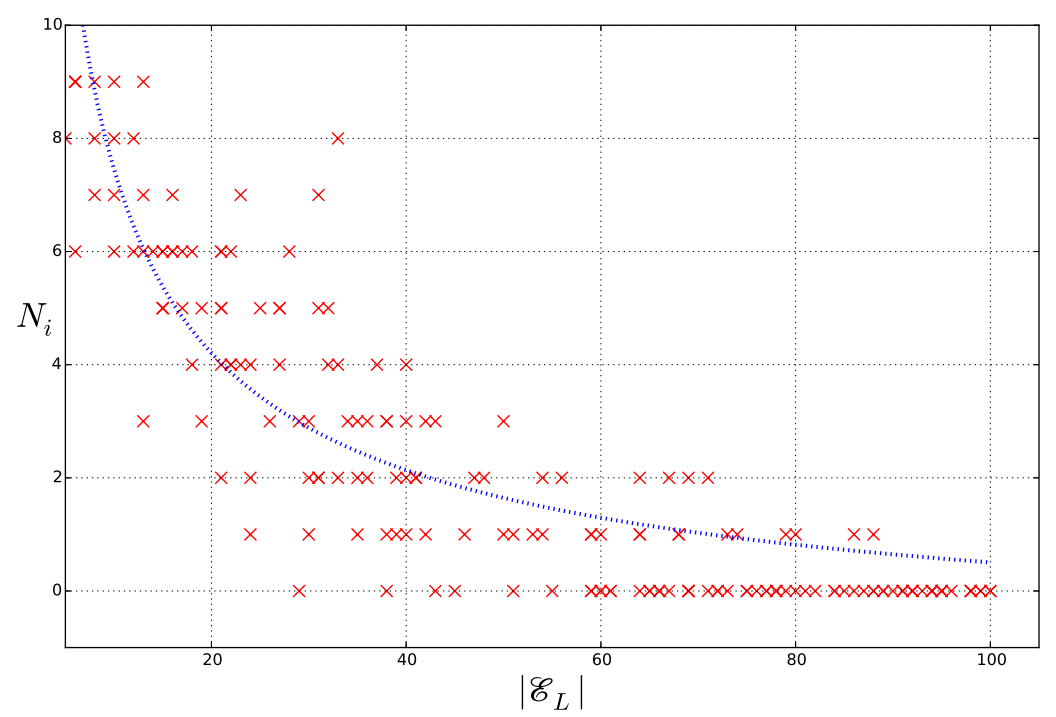

Figure 10: Numerical results for the random simulation of 200 PCR networks. The red crosses show that the number $N_{i}$ of isolated panic nodes roughly decreases as the density $\left|\mathscr{E}_{l}\right|$ of linear connections increases. Indeed, a low number a linear edges can be sufficient to evacuate the panic nodes. The cloud of points is approximated by the blue dotted curve, given by an inverse power law of the type $N_{i}=\frac{k_{1}}{\left|\mathscr{E}_{l}\right|^{\nu}}-k_{2}$, with positive constants $k_{1}, k_{2}$ and $\nu$.

is lower than the density $P_{L}$ of individuals in panic behavior after the same time $T$, in the PCR network obtained by deleting the quadratic couplings. As mentioned before, this indicates the possible benefit of imitation phenomena, when evacuation is made difficult by the geographical relief of the impacted zone.

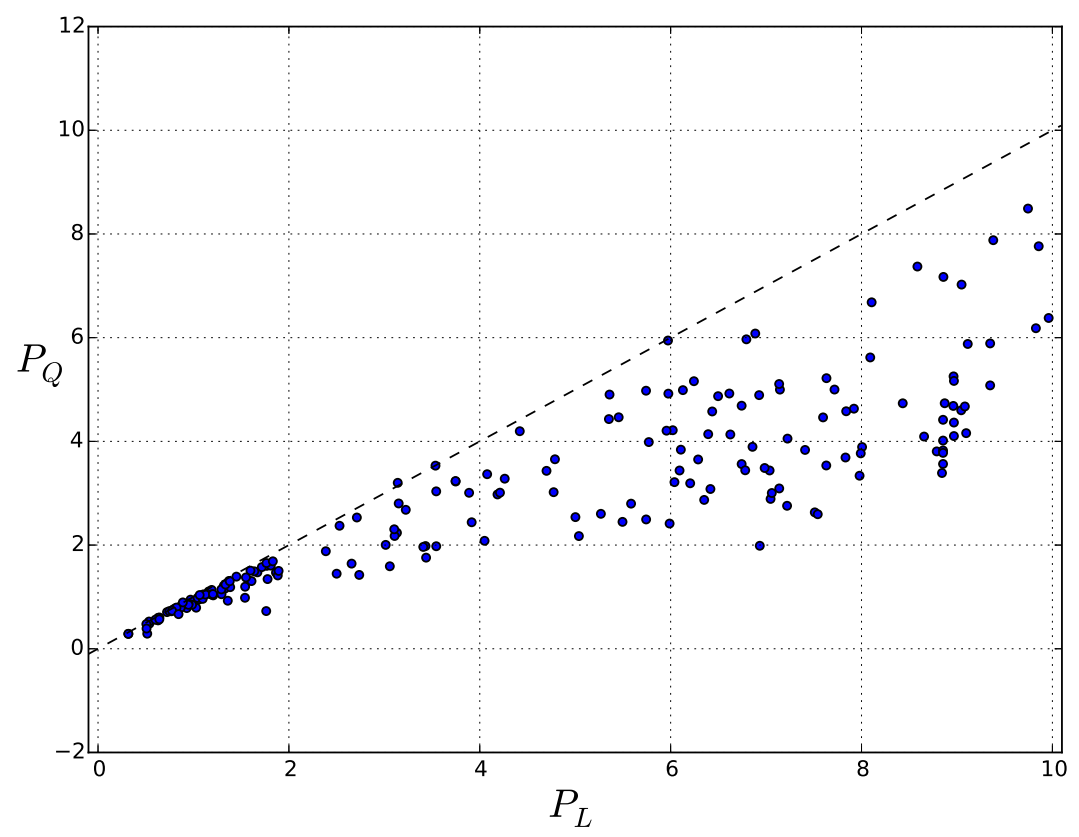

Figure 11: Numerical results for the random simulation of 200 PCR networks. The quadratic connections of panic nodes towards control nodes reduce the panic persistence. After a given time $T$, the density $P_{Q}$ of individuals in panic behavior is lower than the density $P_{L}$ of individuals in panic behavior in the PCR network obtained by deleting the quadratic couplings. 


\section{$\S 5$ Conclusion and perspectives}

In this work, we have studied non identical coupled networks of PCR systems, built with a superposition of linear and quadratic couplings. Patterns emerging from basic two-nodes networks show the decisive role of individuals displacements from panic nodes towards control nodes. A detailed analysis of general abstract networks reveals a necessary and sufficient condition for synchronization towards the trivial equilibrium, that is, evacuation of panic nodes towards control nodes by a directed chain of linear connections. In that case, the global stability of the trivial equilibrium is proved using an extension of Lyapunov's theorem, namely, the LaSalle invariance principle.

Those qualitative results are illustrated by numerical simulations, and represent a new step in the validation of the PCR system, as a mathematical model for understanding and predicting human behaviors during catastrophic events.

In a forthcoming paper, we will study another improvement of the modeling, taking into account some local diffusion phenomena, bringing us to the analysis of a semi-linear parabolic problem.

\section{References}

[1] P. Arena, R. Caponetto, L. Fortuna, A. Rizzo, and M. La Rosa. Self-organization in nonrecurrent complex systems. International Journal of Bifurcation and Chaos, 10(05):1115-1125, 2000.

[2] A. Arenas, A. Díaz-Guilera, J. Kurths, Y. Moreno, and C. Zhou. Synchronization in complex networks. Physics Reports, 469(3):93-153, 2008.

[3] M. Aziz-Alaoui. Synchronization of chaos. Encyclopedia of Mathematical Physics, 26(10):213-226, 2006.

[4] I. Belykh, M. Hasler, M. Lauret, and H. Nijmeijer. Synchronization and graph topology. International Journal of Bifurcation and Chaos, 15(11):3423-3433, 2005.

[5] G. Cantin, N. Verdière, V. Lanza, M. Aziz-Alaoui, R. Charrier, C. Bertelle, D. Provitolo, and E. DubosPaillard. Mathematical modeling of human behaviors during catastrophic events: Stability and bifurcations. International Journal of Bifurcation and Chaos, 26(10):1630025, 2016.

[6] G. Cantin, N. Verdière, V. Lanza, M. Aziz-Alaoui, R. Charrier, C. Bertelle, D. Provitolo, and E. Dubos-Paillard. Control of panic behavior in a non identical network coupled with a geographical model. In PhysCon 2017, pages 1-6, University, Firenze, 2017.

[7] L. Chua. Local activity is the origin of complexity. International journal of bifurcation and chaos, 15(11):3435$3456,2005$.

[8] L. Chua, L. Kocarev, K. Eckert, and M. Itoh. Experimental chaos synchronization in Chua's circuit. International Journal of Bifurcation and Chaos, 02(03):705-708, 1992.

[9] E. Coddington and N. Levinson. Theory of ordinary differential equations. Tata McGraw-Hill Education, 1955.

[10] E. Dijkstra. A note on two problems in connexion with graphs. Numerische mathematik, pages 268-271, 1959.

[11] G. Gallo and S. Pallottino. Shortest path algorithms. Annals of Operations Research, 13(1):1-79, 1988.

[12] M. Golubitsky and I. Stewart. Nonlinear dynamics of networks: the groupoid formalism. Bulletin of the american mathematical society, 43(3):305-364, 2006.

[13] H. Haken. Information and Self-organization: A Macroscopic Approach to Complex Systems. Springer, New York, 2000

[14] A. Korobeinikov and G. Wake. Lyapunov functions and global stability for SIR, SIRS, and SIS epidemiological models. Applied Mathematics Letters, 15(8):955-960, 2002.

[15] N. Krasovskij. Stability of motion: applications of Lyapunov's second method to differential systems and equations with delay. University Press, 1963.

[16] Y. Kuznetsov. Elements of Applied Bifurcation Theory. Applied Mathematical Sciences. Springer New York, 2004.

[17] J. Langa, J. Robinson, and A. Suárez. Stability, instability, and bifurcation phenomena in non-autonomous differential equations. Nonlinearity, 15(3):887, 2002.

[18] J. LaSalle. Some extensions of Liapunov's second method. IRE Transactions on circuit theory, 7(4):520-527, 1960.

[19] L. Pecora and T. Carroll. Master stability functions for synchronized coupled systems. Physical Review Letters, 80(10):2109, 1998.

[20] I. Prigogine and G. Nicolis. Self organization in non-equilibrium systems. J. Wiley and Sons, New York, 1977.

[21] D. Provitolo. Un exemple d'effets de dominos : la panique dans les catastrophes urbaines. Cybergeo: European Journal of Geography, 2005.

[22] D. Provitolo, E. Dubos-Paillard, N. Verdière, V. Lanza, R. Charrier, C. Bertelle, and M. Aziz-Alaoui. Les comportements humains en situation de catastrophe : de l'observation à la modélisation conceptuelle et mathématique. Cybergeo: European Journal of Geography, 735, 2015.

[23] B. Rink and J. Sanders. Coupled cell networks: semigroups, Lie algebras and normal forms. Transactions of the American Mathematical Society, 367(5):3509-3548, 2015. 
[24] E. Steur, W. Michiels, H. Huijberts, and H. Nijmeijer. Networks of diffusively time-delay coupled systems: Conditions for synchronization and its relation to the network topology. Physica D: Nonlinear Phenomena, $277: 22-39,2014$.

[25] H. Thieme. Mathematics in population biology. Princeton University Press, Princeton, New Jersey, 2003.

[26] N. Verdière, G. Cantin, D. Provitolo, V. Lanza, E. Dubos-Paillard, R. Charrier, M. Aziz-Alaoui, and C. Bertelle. Understanding and simulation of human behaviors in areas affected by disasters: From the observation to the conception of a mathematical model. Global Journal of Human Social Science, 15:7-15, 2015.

[27] N. Verdière, V. Lanza, R. Charrier, E. Dubos-Paillard, C. Bertelle, and M. Aziz-Alaoui. Mathematical modeling of human behaviors during catastrophic events. In ICCSA 2014, pages 1-30, University, Le Havre, 2014.

[28] F. Verhulst. Nonlinear differential equations and dynamical systems. Springer Science \& Business Media, 1996.

[29] C. Zhang, B. Zheng, and L. Wang. Multiple Hopf bifurcations of three coupled van der pol oscillators with delay. Applied Mathematics and Computation, 217(17):7155-7166, 2011.

[30] C. Zhang and L. Zheng, B.and Wang. Multiple Hopf bifurcations of symmetric bam neural network model with delay. Applied Mathematics Letters, 22(4):616-622, 2009. 